\section{National accounts}

\section{National accounts}

\section{(Tables 16.1 to 16.22 )}

The tables which follow are based on those in the Blue Book 2008 Edition. Some of the figures are provisional and may be revised later; this applies particularly to the figures for 2006 and 2007.

The accounts are based on the European System of Accounts 1995 (ESA95). The Blue Book contains an introduction to the system of the UK accounts outlining some of the main concepts and principles of measurement used. It explains how key economic indicators are derived from the sequence of accounts and how the figures describing the whole economy are broken-down by sector and by industry. A detailed description of the structure for the accounts is provided in a separate Office for National Statistics publication United Kingdom National Accounts: Concepts, Sources and Methods (TSO 1998). Further information on the financial accounts is given in the Financial Statistics Explanatory Handbook.

In the tables in this chapter on national income, analyses by industry are based, as far as possible, on the Standard Industrial Classification Revised 2003. The principal aggregate measured in these tables is the Gross Domestic Product (GDP). This is a concept of the value of the total economic activity taking place in UK territory. It can be viewed as incomes earned, as expenditures incurred, or as production. Adding all primary incomes received from the rest of the world and deducting all primary incomes payable to non-residents produces Gross National Income (GNI) (previously known as Gross National Product). This is a concept of the value of all incomes earned by UK residents.

ESA95, the internationally compatible accounting framework, provides a systematic and detailed description of the UK economy. It includes the sector accounts which provide, by institutional sector, a description of the different stages of the economic process from production through income generation, distribution and use of income to capital accumulation and financing; and the input-output framework, which describes the production process in more detail. It contains all the elements required to compile such aggregate measures as GDP, GNI and saving.

\section{Gross Domestic Product and Gross National Income}

\author{
(Tables 16.1, 16.2, 16.3)
}

Table 16.1 shows the main national accounts aggregates, both at current prices and chained volume measures.

Table 16.2 shows the various money flows which generate the GDP and GNI. The output approach to GDP shows the total output of goods and services, the use of goods and services in the production process (intermediate consumption) and taxes and subsidies on products. The expenditure approach to GDP shows consumption expenditure by households and government, gross capital formation and expenditure on UK exports by overseas purchasers. The sum of these items overstates the amount of income generated in the UK by the value of imports of goods and services. This item is therefore subtracted to produce GDP at market prices.

The income approach to GDP shows gross operating surplus, mixed income and compensation of employees (previously known as income from employment). Taxes are added and subsidies are deducted to produce the total of the income based components at market prices.

Table 16.2 also shows the primary incomes received from the rest of the world, which are added to GDP, and primary incomes payable to non-residents, which are deducted from GDP, to arrive at GNI. Primary income comprises compensation of employees, taxes less subsidies on production, and property and entrepreneurial income.

Table 16.3 shows the expenditure approach to the chained volume measure of GDP. When looking at the change in the economy over time the main concern is usually whether more goods and services are actually being produced now than at some time in the past. Over time changes in current price GDP show changes in the monetary value of the components of GDP and, as these changes in value can reflect changes in both price and volume, it is difficult to establish how much of an increase in the series is due either to increased activity in the economy or to an increase in the price level. As a result, when looking at the real growth in the economy over time, it is useful to look at volume estimates of GDP. In chained volume series, volume measures for each year are produced in prices of the previous year. These volume measures are then 'chain-linked' together to produce a continuous time series.

\section{Industrial analysis}

(Tables 16.4, 16.5)

The analysis of gross value added by industry at current prices shown in Table 16.4 reflects the estimates based on the 
Standard Industrial Classification, revised 2003 (SIC2003). The table is based on current price data reconciled through the input-output process for 1992 to 2006 . The estimates are valued at basic prices, that is, the only taxes included in the price will be taxes paid as part of the production process, such as business rates, and not any taxes specifically levied on the production of a unit of output, for example VAT. Table 16.5 shows chained volume measures of gross value added at basic prices by industry. Chained volume measures of gross value added (output approach) provides the lead indicator of economic change in the short term. The output analysis of gross value added is estimated in terms of change and expressed in index number form. It is therefore inappropriate to show as a statistical adjustment any divergence of an output measure of GDP derived from other measures of GDP. Such an adjustment does, however, exist implicitly.

\section{Sector analysis - Distribution of income accounts and capital account}

\section{(Tables 16.6 to 16.13 )}

The National Accounts accounting framework includes the sector accounts which provide, by institutional sector, a description of the different stages of the economic process, from production through income generation, distribution and use of income to capital accumulation and financing

Tables 16.6 to 16.12 show the allocation of primary income account and the secondary distribution of income account for the non-financial corporations, financial corporations, government and households sectors. Additionally, Table 16.12 shows the use of income account for the households sector and Table 16.13 provides a summary of the capital account. The full sequence of accounts is shown in the Blue Book.

The allocation of primary income account shows the resident units and institutional sectors as recipients rather than producers of primary income. It demonstrates the extent to which operating surpluses are distributed to the owners of the enterprises. The resources side of the allocation of primary income accounts includes the components of the income approach to measurement of GDP. The balance of this account is the gross balance of primary income (B. $5 \mathrm{~g}$ ) for each sector, and if the gross balance is aggregated across all sectors of the economy the result is Gross National Income.

The secondary distribution of income account describes how the balance of income for each sector is allocated by redistribution; through transfers such as taxes on income, social contributions and benefits and other current transfers. The balancing item of this account is Gross Disposable Income (GDI) (B.6g). For the households sector, the chained volume measure of GDI is shown as real household disposable income.
Table 16.12 shows, for the households sector, the use of disposable income where the balancing item is saving (B.8g). For the non-financial corporations sector the balancing item of the secondary distribution of income account, gross disposable income (B.6g) is equal to saving (B.8g).

The summary capital account (Table 16.13) brings together the saving and investment of the several sectors of the economy. It shows saving, capital transfers, gross capital formation and net acquisition of non-financial assets for each of the four sectors.

\section{Household and non-profit institutions serving households consumption expenditure at current market prices and chained volume measures}

\section{(Tables 16.14 to 16.17 )}

Household and non-profit institutions serving households (NPISH) consumption expenditure is a major component of the expenditure measure of GDP, both at current prices (Table 16.2) and chained volume measures (Table 16.3).

Household final consumption expenditure includes the value of income-in-kind and imputed rent of owner-occupied dwellings, but excludes business expenditure allowed as deductions in computing income for tax purposes. It includes expenditure on durable goods, for instance motor cars, which from the point of view of the individual might more appropriately be treated as capital expenditure. The only exceptions are the purchase of land and dwellings and costs incurred in connection with the transfer of their ownership and expenditure on major improvements by occupiers, which are treated as personal capital expenditure.

The estimates of household consumption expenditure include purchases of second-hand as well as new goods, less the proceeds of sales of used goods.

The most detailed figures are published quarterly in Consumer Trends (available as a web-only publication on the Office for National Statistics website: www.statistics.gov.uk).

\section{Change in inventories (previously known as value of physical increase in stocks and work in progress)}

\section{(Table 16.18)}

This table gives a broad analysis by industry and, for manufacturing industry, by asset, of the value of entries less withdrawals and losses of inventories (stocks). 
National accounts

\section{Gross fixed capital formation}

(Table 16.19 to 16.22 )

Gross fixed capital formation comprises expenditure on the replacement of, and additions to, fixed capital assets located in the UK, including all ships and aircraft of UK ownership. 
National accounts

\section{United Kingdom national and domestic product ${ }^{1}$ \\ Main aggregates}

At current prices and chained volume measures, reference year 2003

Indices $(2003=100)$ and £ million

\begin{tabular}{lllllllll}
2000 & 2001 & 2002 & 2003 & 2004 & 2005 & 2006 & 2007 & 2008 \\
\hline
\end{tabular}

INDICES $(2003=100)$

VALUES AT CURRENT PRICES

Gross domestic product at current market prices

("money GDP")

Gross value added at current basic prices

YBEX $\quad 85$.

89.7

94.7

$94.4 \quad 100.0$

100.0
100.0

105.3

105.3

109.9

$\begin{array}{lll} & 116.9 & 122.9\end{array}$

116.0

122.9

126.6

CHAINED VOLUME MEASURES

Gross domestic product at market prices

Gross national disposable income at market prices

Gross value added at basic prices

$\begin{array}{llllllllll}\text { YBEZ } & 93.0 & 95.3 & 97.3 & 100.0 & 102.8 & 104.9 & 107.8 & 111.1 & 111.9\end{array}$

$\begin{array}{llllllllll}\text { YBFP } & 90.7 & 93.9 & 97.1 & 100.0 & 102.8 & 104.2 & 106.1 & 110.6 & 112.7\end{array}$

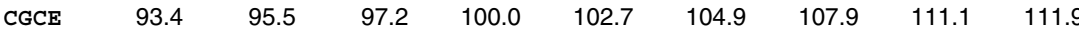

PRICES

Implied deflator of GDP at market prices

YBGB

$92.2 \quad 94.1$

97.0

100.0

102.5

104.8

107.5

110.6

113.1

VALUES AT CURRENT PRICES (£ million)

Gross measures (before deduction of fixed capital consumption) at current market prices

Gross Domestic Product ("money GDP")

Employment, property and entrepreneurial income from the rest of the world (receipts less payments)

Subsidies (receipts) less taxes (payments) on products from/to the rest of the world

Other subsidies on production from/to the rest of the world - $-\mathrm{ZZOZ}$

YBHA

97653310218281075564113974612005951252505132186014005261442921

$\begin{array}{lllllllll}\text { YBGG } & 1962 & 9425 & 18286 & 17523 & 17830 & 21872 & 10097 & 8606\end{array}$

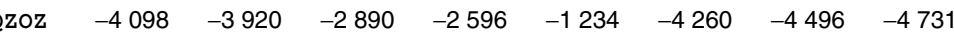

Gross National Income (GNI)

Current transfers from the rest of the world (receipts less payments)

Gross National Disposable Income

ABMX 97473210279151091479115526512177831273525133068114193191474243

$\begin{array}{lllllllll}- \text { YBGF } & -6016 & -3182 & -6500 & -7843 & -9645 & -11052 & -10657 & -11943\end{array}$

NQCO 96871610247331084979114742212081381262473132002414074591462424

Adjustment to current basic prices

Gross Domestic Product (at current market prices)

Adjustment to current basic prices (less taxes plus subsidies on products)

Gross Value Added (at current basic prices)

-NQBU $-112248-114234-118470-124738-132362-137347-144658-153147-148642$

ABML $\quad 864285907594957094101500810685741115121117723212473791294279$

Net measures (after deduction of fixed

capital consumption) at current market prices

Net domestic product

Net national income

Net national disposable income

$\begin{array}{llllll}N H R K & 865282 & 906032 & 95365010141431065411111398511740021242899\end{array}$

NSRX $\quad 863481 \quad 91211996956510296621082599113500511828231249717$

NQCP $\quad 857465 \quad 90893796306510218191072954112395311721661237774$

CHAINED VOLUME MEASURES

(Reference year 2003, \& million)

Gross measures (before deduction of fixed capital consumption) at market prices

Gross Domestic Product

Terms of trade effect ("Trading gain or loss")

Real gross domestic income

Real employment, property and entrepreneurial income from the rest of the world (receipts less payments)

Subsidies (receipts) less taxes (payments) on production from/to the rest of the world

Other subsidies on production from/to the rest of the world -

Gross National Income (GNI)

Real current transfers from the rest of the world (receipts less payments)

Gross National Disposable Income

Adjustment to basic prices

Gross Domestic Product (at market prices)

Adjustment to basic prices

(less taxes plus subsidies on products)

Gross Value Added (at basic prices)

Net measures (after deduction of fixed capital consumption) at market prices

Net national income at market prices

Net national disposable income at market prices

1 See chapter text
ABMI 105965810857451108508113974611711781195276122919612663471275299

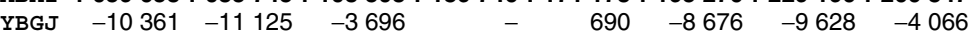

YBGL 10492971074620110481211397461171868118660012195681262331

$\begin{array}{lllllllll}\text { YBGI } & 2103 & 9901 & 18775 & 17523 & 17411 & 20732 & 9323 & 7768\end{array}$

$\begin{array}{lllllllll}\text { OZPB } & -4392 & -4118 & -2967 & -2596 & -1205 & -4038 & -4151 & -4270\end{array}$

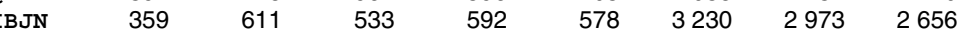

YBGM 10473371081003112115411552651188652120652412277131268485

$\begin{array}{lllllllll}\text {-YBGP } & -6448 & -3342 & -6673 & -7843 & -9419 & -10476 & -9840 & -10779\end{array}$

YBGO 104088510776651114481114742211792331196048121787312687121293408

ABMI 105965810857451108508113974611711781195276122919612663471275299

-NTAQ $-112020-116584-121657-124738-128532-130433-133633-138328$

AвмM 947927969279986849101500810426461064843109556311275741135940

-CIHA $-114678-118027-123357-125508-133307-134030-139661-143385$

YBET $\quad 932606 \quad 962937 \quad 99772410297121055449107281810884861123509$

YBEY 92615095961399105310218691046030106234210786461112730 


\section{United Kingdom gross domestic product and national income ${ }^{1}$ Current prices}

$£$ million

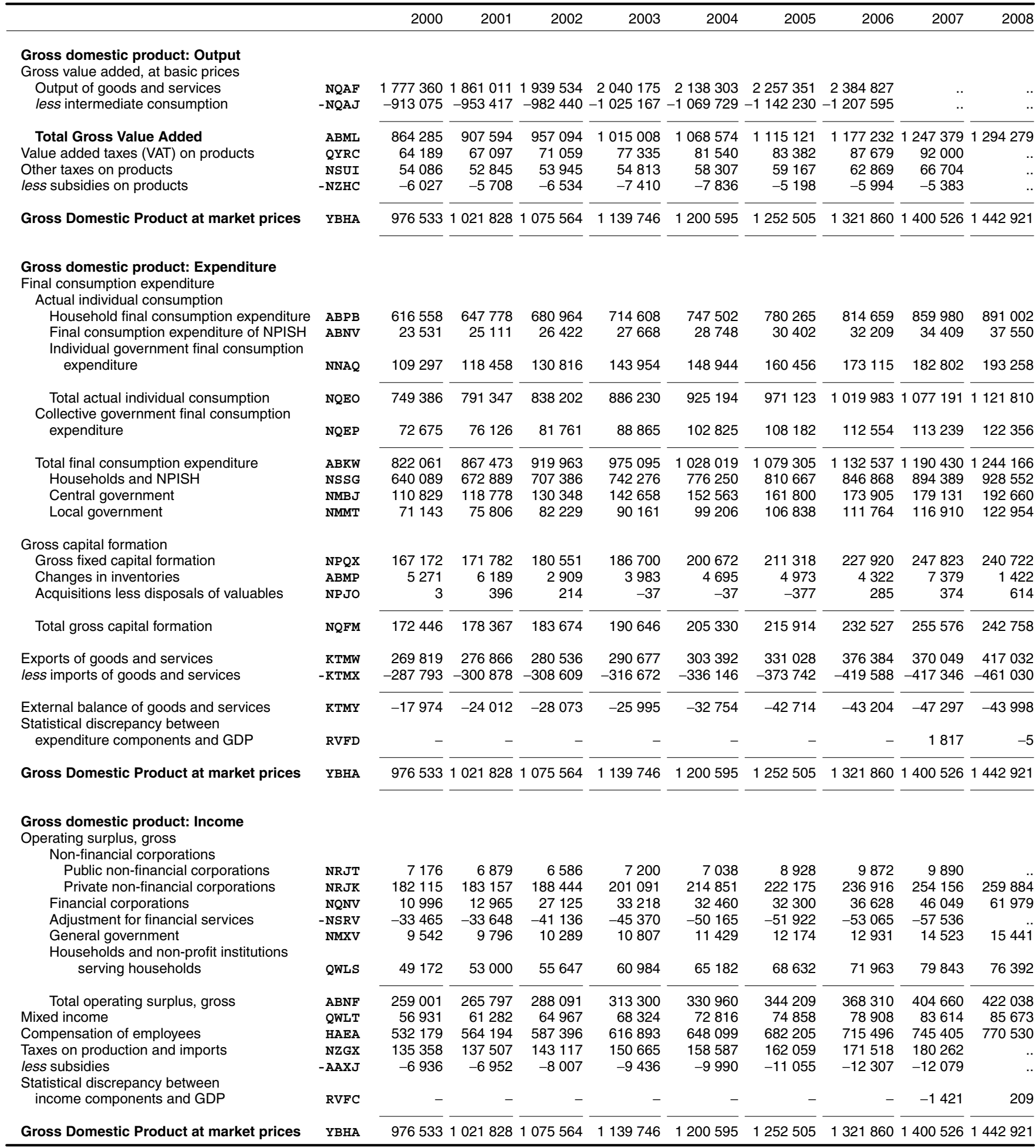




\section{United Kingdom gross domestic product and national income ${ }^{1}$

\begin{tabular}{|c|c|c|c|c|c|c|c|c|c|c|}
\hline & & 2000 & 2001 & 2002 & 2003 & 2004 & 2005 & 2006 & 2007 & 2008 \\
\hline $\begin{array}{l}\text { Gross Domestic Product at market prices } \\
\text { Compensation of employees }\end{array}$ & YBHA & 976533 & 1021828 & 1075564 & 1139746 & 1200595 & 1252505 & 1321860 & 1400526 & 1442921 \\
\hline receipts from the rest of the world & KTMN & 1032 & 1087 & 1121 & 1116 & 931 & 974 & 1058 & 1159 & 1221 \\
\hline less payments to the rest of the world & -КTMO & -882 & -1021 & -1054 & -1057 & -1425 & -1584 & -1803 & -1824 & -1840 \\
\hline Total & KTMP & 150 & 66 & 67 & 59 & -494 & -610 & -745 & -665 & -619 \\
\hline less Taxes on products paid to the rest of the world & & & & & & & & & & \\
\hline Other subsidies on production & $-\mathrm{IBJL}$ & 292 & 298 & 519 & .. & .. & .. & .. & .. & . \\
\hline Property and entrepreneurial income & & & & & & & & & & \\
\hline receipts from the rest of the world & HMBN & 131902 & 137447 & 120543 & 122069 & 137382 & 185765 & 237448 & 290399 & 271299 \\
\hline less payments to the rest of the world & -HMBO & -130090 & -128088 & -102324 & -104605 & -119058 & -163283 & -226606 & -269162 & -237551 \\
\hline Total & HMBM & 1812 & 9359 & 18219 & 17464 & 18324 & 22482 & 10842 & 21237 & 33748 \\
\hline Gross National Income at market prices & ABMX & 974732 & 1027915 & 1091479 & 1155265 & 1217783 & 31273525 & 51330681 & 1419319 & 1474243 \\
\hline
\end{tabular}

\section{United Kingdom gross domestic product ${ }^{1}$ Chained volume measures, reference year 2003}

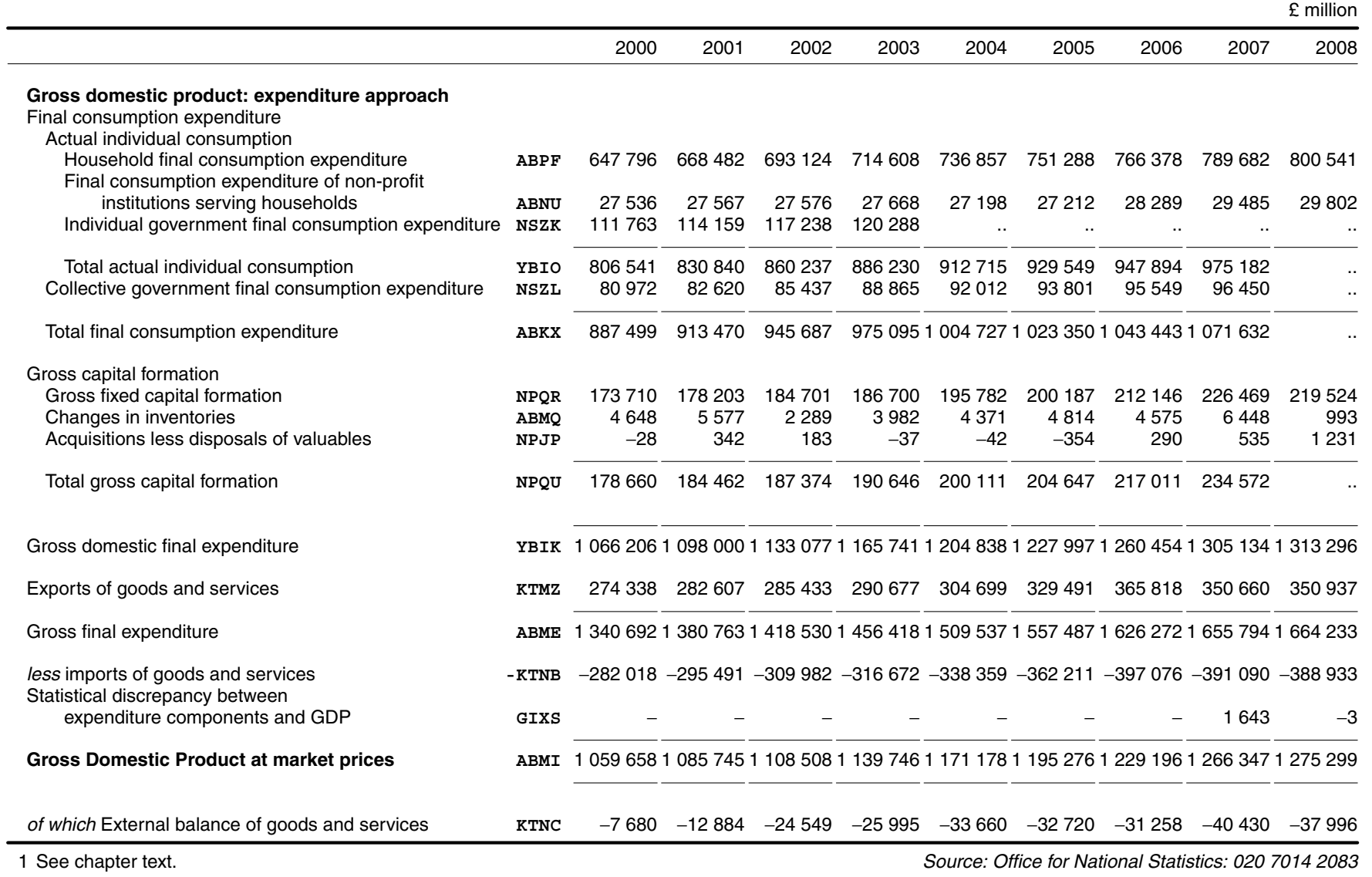




\begin{tabular}{llllllllllll}
\hline & & 2000 & 2001 & 2002 & 2003 & 2004 & 2005 & 2006 & 2007 & 2008 \\
\hline Agriculture, hunting, forestry and fishing & EWSH & 8532 & 8333 & 9007 & 9806 & 10600 & 7422 & 7865 &.. &..
\end{tabular}

Production

Mining and quarrying

Mining and quarrying of energy producing materials

Mining of coal

Extraction of mineral oil and natural gas

Other mining and quarrying

Total mining and quarrying

Manufacturing

Food; beverages and tobacco

Textiles and textile products

Leather and leather products

Wood and wood products

Pulp, paper and paper products; publishing and printing

Coke, petroleum products and nuclear fuel

Chemicals, chemical products and man-made fibres

Rubber and plastic products

Other non-metal mineral products

Basic metals and fabricated metal products

Machinery and equipment not elsewhere classified

Electrical and optical equipment

Transport equipment

Manufacturing not elsewhere classified

Total manufacturing

Electricity, gas and water supply

Total production

Construction

\begin{tabular}{|c|c|c|c|c|c|c|c|c|}
\hline $\begin{array}{l}\text { QTOQ } \\
\text { QTOR } \\
\text { QTOS }\end{array}$ & $\begin{array}{r}607 \\
22174 \\
1784\end{array}$ & $\begin{array}{r}545 \\
20825 \\
1750\end{array}$ & $\begin{array}{r}538 \\
19911 \\
1469\end{array}$ & $\begin{array}{r}472 \\
19451 \\
1519\end{array}$ & $\begin{array}{r}398 \\
20321 \\
1841\end{array}$ & $\begin{array}{r}323 \\
25265 \\
2081\end{array}$ & $\begin{array}{r}314 \\
29776 \\
2112\end{array}$ & $\begin{array}{r}309 \\
29831 \\
2291\end{array}$ \\
\hline EWSL & 24565 & 23120 & 21918 & 21442 & 22560 & 27669 & 32202 & .. \\
\hline QTOU & 19963 & 20655 & 20834 & 21408 & 21979 & 21826 & 22143 & 22996 \\
\hline QTOV & 5813 & 5343 & 4818 & 4282 & 4240 & З 977 & 3944 & 3887 \\
\hline QTOW & 747 & 645 & 590 & 462 & 474 & 424 & 418 & 429 \\
\hline QTOX & 2294 & 2332 & 2479 & 2655 & 2790 & 3052 & 3200 & 3603 \\
\hline QTOY & 20187 & 20129 & 20008 & 19780 & 19378 & 19315 & 19246 & 19530 \\
\hline QTOZ & 2336 & 2488 & 2435 & 2377 & 2439 & 2529 & 2506 & 2636 \\
\hline QTPA & 15040 & 16077 & 16083 & 16149 & 17321 & 17246 & 18847 & 18874 \\
\hline QTPB & 7609 & 7656 & 7569 & 7516 & 7380 & 7563 & 7928 & 7929 \\
\hline QTPC & 4965 & 5033 & 5296 & 5417 & 5528 & 5193 & 5469 & 5718 \\
\hline QTPD & 15903 & 15525 & 14897 & 14774 & 15678 & 16402 & 16400 & 17312 \\
\hline QTPE & 12346 & 12256 & 12085 & 12146 & 12381 & 12525 & 13901 & 14876 \\
\hline QTPF & 20337 & 18347 & 16468 & 15545 & 15661 & 16117 & 15908 & 15730 \\
\hline QTPG & 15987 & 16091 & 16178 & 15903 & 15652 & 15932 & 16305 & 16805 \\
\hline QTPH & 6477 & 6643 & 6567 & 6429 & 6361 & 6491 & 6938 & 7403 \\
\hline EWSP & 150009 & 149223 & 146308 & 144845 & 147261 & 148591 & 153155 & .. \\
\hline EWST & 15798 & 15660 & 16052 & 16405 & 16672 & 16667 & 18870 & .. \\
\hline QTPK & 190367 & 188000 & 184277 & 182690 & 186494 & 192928 & 204225 & 208855 \\
\hline EWSX & 45626 & 50526 & 54684 & 59522 & 63955 & 68019 & 73993 &.. \\
\hline
\end{tabular}

Service industries

Wholesale and retail trade (including motor trade)

repair of motor vehicles, personal and household goods

Hotels and restaurants

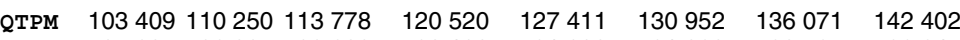

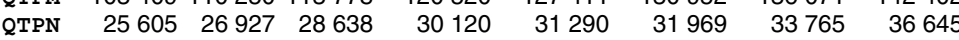

Transport, storage and communication

Transport and storage

Communication

Total

Financial intermediation

Adjustment for financial services (FISIM)

$\begin{array}{lllllllll}\text { QTPO } & 42476 & 43184 & 44501 & 47022 & 49576 & 50203 & 50491 & 53434\end{array}$

$\begin{array}{lllllllll}\text { QTPP } & 26726 & 27317 & 28562 & 29566 & 30253 & 30307 & 30537 & 31892\end{array}$

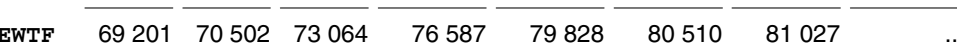

$\begin{array}{lrlllllll}\text { QTPR } & 44990 & 48202 & 63368 & 71530 & 75042 & 79356 & 91011 & 95398\end{array}$

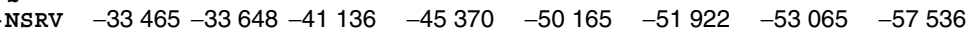

Real estate, renting and business activities

Letting of dwellings including imputed rent

of owner occupiers

Other real estate, renting and business activities

Total

Public administration and defence (PAD)

Education

Health and social work

Other social and personal services,

private households with employees

and extra-territorial organisations

Total service industries

\begin{tabular}{|c|c|c|c|c|c|c|c|c|}
\hline $\begin{array}{l}\text { QTPS } \\
\text { QTPT }\end{array}$ & $\begin{array}{r}57261 \\
131098\end{array}$ & $\begin{array}{r}61352 \\
142688\end{array}$ & $\begin{array}{r}64249 \\
150598\end{array}$ & $\begin{array}{r}69298 \\
162906\end{array}$ & $\begin{array}{r}76166 \\
170882\end{array}$ & $\begin{array}{r}80155 \\
179180\end{array}$ & $\begin{array}{r}84809 \\
188835\end{array}$ & $\begin{array}{r}90065 \\
212540\end{array}$ \\
\hline QTPU & 188359 & 204040 & 214847 & 232204 & 247048 & 259335 & 273644 & 302605 \\
\hline $\begin{array}{l}\text { EWTN } \\
\text { QTPW } \\
\text { QTPX }\end{array}$ & $\begin{array}{l}42712 \\
48111 \\
55282\end{array}$ & $\begin{array}{l}45025 \\
51675 \\
59549\end{array}$ & $\begin{array}{l}47528 \\
55099 \\
64492\end{array}$ & $\begin{array}{l}51302 \\
58328 \\
70593\end{array}$ & $\begin{array}{l}55393 \\
61814 \\
74417\end{array}$ & $\begin{array}{ll}60 & 096 \\
66 & 186 \\
78 & 497\end{array}$ & $\begin{array}{l}63033 \\
69345 \\
81883\end{array}$ & $\begin{array}{l}73105 \\
86689\end{array}$ \\
\hline NTV & 42086 & 44561 & 48312 & 51804 & 55283 & 59852 & 61368 & .. \\
\hline תחי & 619756 & 660729 & 09122 & 762988 & 807529 & 846753 & 891148 & 950333 \\
\hline
\end{tabular}

All industries

ABML 864285907594957094101500810685741115121117723212473791294279

1 See chapter text. Components may not sum to totals as a result of round-

ing.

2 Because of differences in the annual and monthly production inquiries, estimates of current price output and value added by industry derived from the current price input-output supply-use balances are not consistent with the equivalent measures of constant price growth given in Table 16.5. These differences do not affect GDP totals. For further information see "Experimental Constant Price Input-Output Supply-Use Balances: An approach to improving the quality of the national accounts" Nadim Ahmad, Economic Trends, July 1999 (No. 548) 


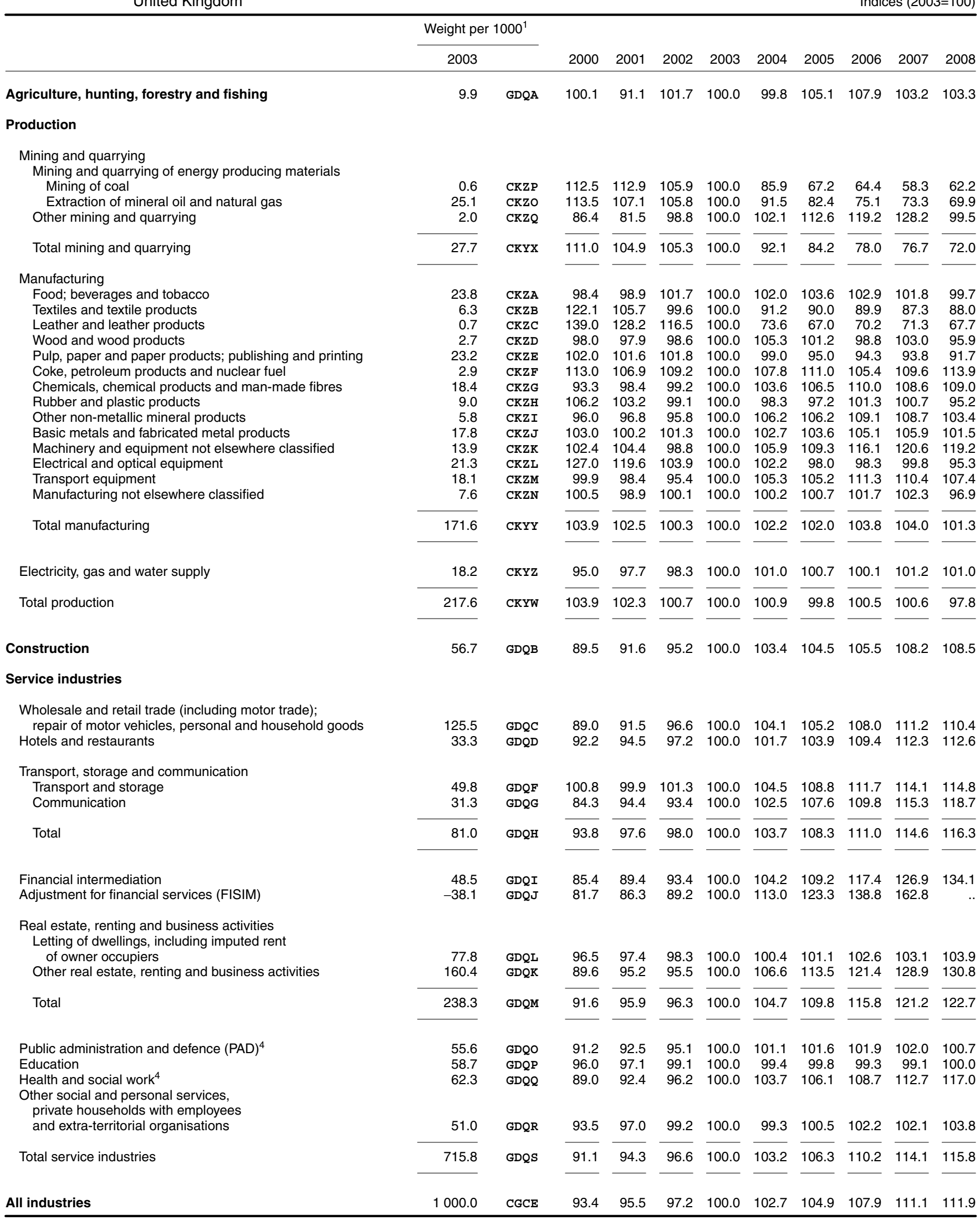

\footnotetext{
1 See chapter text. The weights are in proportion to total gross value added 3 See footnote 2 to Table 16.4.
}

(GVA) in 2003 and are used to combine the industry output indices to cal- 4 The GVA for PAD, education and Health and social work in this table follows culate the totals for 2004 and 2005. For 2003 and earlier, totals are calculated using the equivalent weight for the previous year (eg totals for 2002 use 2001 weights).

2 As GVA is expressed in index number form, it is inappropriate to show as a statistical adjustment any divergence from the other measures of GDP. Such an adjustment does, however, exist implicitly. the SIC(92) and differs from that used in Table 2.3 inUnited Kingdom National Accounts (the Blue Book) which is based on Input-Output groups. The administration costs of the NHS are included in PAD in this table but are included in Health and social work in Table 2.3.

Source: Office for National Statistics: 02070142083 


\begin{tabular}{|c|c|c|c|c|c|c|c|c|c|c|}
\hline & & 2000 & 2001 & 2002 & 2003 & 2004 & 2005 & 2006 & 2007 & 2008 \\
\hline \multicolumn{11}{|l|}{ Resources } \\
\hline Operating surplus, gross & NQBE & 189291 & 190036 & 195030 & 208291 & 221889 & 231103 & 246788 & 264245 & 268226 \\
\hline Distributed income of corporations & EABD & 26631 & 36868 & 32210 & & & & & & \\
\hline Reinvested earnings on direct foreign investment & HDVR & 20118 & 22950 & 26893 & 12492 & 22713 & 33199 & 36426 & 49474 & 50296 \\
\hline Attributed property income of insurance policy-holders & FAOF & 489 & 280 & 302 & .. & .. & .. & .. & .. & .. \\
\hline Rent & FAOG & 117 & 117 & 118 & .. & .. & .. & .. & .. & .. \\
\hline Total & FAKY & 61667 & 74102 & 67229 & 73070 & 80467 & 98171 & 106500 & 118886 & 110554 \\
\hline Total resources & FBXJ & 250958 & 264138 & 262259 & 281361 & 302356 & 329274 & 353288 & 383131 & 378780 \\
\hline \multicolumn{11}{|l|}{$\begin{array}{l}\text { Uses } \\
\text { Property income, paid }\end{array}$} \\
\hline Interest & EABG & 30405 & 30661 & 29045 & 29592 & 34937 & 39338 & 45229 & 55310 & 50455 \\
\hline Distributed income of corporations & NVCS & 83202 & 100810 & 91868 & & & .. & .. & . & \\
\hline Total & FBXK & 123265 & 137950 & 119879 & 128885 & 136074 & 152442 & 168741 & 172402 & 170536 \\
\hline Balance of primary incomes, gross & NQBG & 127693 & 126188 & 142380 & 152476 & 166282 & 176832 & 184547 & 210729 & 208244 \\
\hline Total uses & FBXJ & 250958 & 264138 & 262259 & 281361 & 302356 & 329274 & 353288 & 383131 & 378780 \\
\hline After deduction of fixed capital consumption & $-\mathrm{DBGF}$ & -66420 & -68362 & -70547 & -72598 & -75559 & -77278 & -80360 & -83565 & * \\
\hline Balance of primary incomes, net & FBXQ & 61273 & 57826 & 71833 & 79878 & 90723 & 99554 & 104187 & 113074 & \\
\hline
\end{tabular}

1 See chapter text.

2 Before deduction of fixed capital formation.

\begin{tabular}{|c|c|c|c|c|c|c|c|c|c|c|}
\hline & & 2000 & 2001 & 2002 & 2003 & 2004 & 2005 & 2006 & 2007 & 2008 \\
\hline \multicolumn{11}{|l|}{ Resources } \\
\hline Balance of primary incomes, gross & NQBG & 127693 & 126188 & 142380 & 152476 & 166282 & 176832 & 184547 & 210729 & 208244 \\
\hline \multicolumn{11}{|l|}{ Social contributions } \\
\hline Imputed social contributions & NSTJ & 4175 & 4357 & 4575 & 4229 & 3810 & 3535 & 3425 & 3508 & 4148 \\
\hline \multicolumn{11}{|l|}{$\begin{array}{l}\text { Current transfers other than taxes, } \\
\text { social contributions and benefits }\end{array}$} \\
\hline Non-life insurance claims & FCBP & 4456 & 4565 & 7789 & .. & .. & .. & .. & .. & .. \\
\hline Miscellaneous transfers & NRJY & 622 & 619 & 616 & .. & .. & .. & .. & .. & .. \\
\hline Total & NRJB & 5847 & 3836 & 5543 & 6124 & 6550 & 7261 & 8368 & 4220 & 4930 \\
\hline Total resources & FCBR & 137715 & 134381 & 152498 & 162829 & 176642 & 187628 & 196340 & 218457 & 217322 \\
\hline \multicolumn{11}{|l|}{ Uses } \\
\hline Social benefits other than social transfers in kind & NSTJ & 4175 & 4357 & 4575 & 4229 & 3810 & 3535 & 3425 & 3508 & 4148 \\
\hline \multicolumn{11}{|l|}{$\begin{array}{l}\text { Current transfers other than taxes, } \\
\text { social contributions and benefits }\end{array}$} \\
\hline Net non-life insurance premiums & FCBY & 4456 & 4565 & 7789 & .. & .. & .. & .. & .. & .. \\
\hline Miscellaneous current transfers & FDBI & 413 & 411 & 422 & 434 & 446 & .. & .. & .. & .. \\
\hline $\begin{array}{l}\text { Total, other current transfers } \\
\text { Gross Disposable Income }\end{array}$ & $\begin{array}{l}\text { FCBX } \\
\text { NRJD }\end{array}$ & $\begin{array}{r}6315 \\
102728\end{array}$ & $\begin{array}{r}4220 \\
102627\end{array}$ & $\begin{array}{r}5876 \\
118009\end{array}$ & $\begin{array}{r}6462 \\
128436\end{array}$ & $\begin{array}{r}6973 \\
138491\end{array}$ & $\begin{array}{r}7749 \\
142742\end{array}$ & $\begin{array}{r}8842 \\
146890\end{array}$ & $\begin{array}{r}4708 \\
171972\end{array}$ & $\begin{array}{r}5418 \\
169774\end{array}$ \\
\hline Total uses & FCBR & 137715 & 134381 & 152498 & 162829 & 176642 & 187628 & 196340 & 218457 & 217322 \\
\hline After deduction of fixed capital consumption & $-D B G F$ & -66420 & -68362 & -70547 & -72598 & -75559 & -77278 & -80360 & -83565 & .. \\
\hline & FCCF & 36308 & 34265 & 47462 & 55838 & 62932 & 65464 & 66530 & 74338 & \\
\hline
\end{tabular}


National accounts

\begin{tabular}{|c|c|c|c|c|c|c|c|c|c|c|}
\hline & & 2000 & 2001 & 2002 & 2003 & 2004 & 2005 & 2006 & 2007 & 2008 \\
\hline \multicolumn{11}{|l|}{ Resources } \\
\hline Operating surplus, gross & NMXV & 9542 & 9796 & 10289 & 10807 & 11429 & 12174 & 12931 & 14523 & 15441 \\
\hline \multicolumn{11}{|l|}{$\begin{array}{l}\text { Taxes on production and imports, received } \\
\text { Taxes on products }\end{array}$} \\
\hline Value added tax (VAT) & NZGF & 59998 & 63525 & 68258 & 74603 & 79761 & 81416 & 85591 & 89698 & 88909 \\
\hline \multicolumn{11}{|l|}{ Taxes and duties on imports excluding VAT } \\
\hline Import duties & NMXZ & - & - & - & - & - & - & - & - & \\
\hline Taxes on imports excluding VAT and import duties & NMBT & - & - & - & - & - & .. & .. & . & \\
\hline Total taxes on products & NVCC & 111941 & 114267 & 120252 & 127453 & 135898 & 138322 & 146126 & 154007 & 149268 \\
\hline Other taxes on production & NMYD & 17083 & 17565 & 18113 & 18517 & 18853 & 19706 & 20831 & 21543 & 22347 \\
\hline Total taxes on production and imports, received & NMYE & 129024 & 131832 & 138365 & 145970 & 154751 & 158028 & 166957 & 175550 & 171615 \\
\hline \multicolumn{11}{|l|}{ less Subsidies, paid } \\
\hline Subsidies on products & -NMYF & -3791 & -3953 & -4672 & -5311 & -5111 & -5198 & -5994 & -5591 & -5532 \\
\hline Other subsidies on production & -LIUF & -574 & -662 & -954 & -1434 & -1562 & -2449 & -3093 & -3470 & -3419 \\
\hline \multicolumn{11}{|l|}{ Property income, received } \\
\hline Total Interest & NMYL & 7403 & 7359 & 6683 & 7131 & 6804 & 6458 & 7109 & 8125 & 8170 \\
\hline Distributed income of corporations & NMYM & 5480 & 4710 & 3290 & 3027 & 2763 & 2866 & 2541 & 3126 & 3079 \\
\hline $\begin{array}{l}\text { Property income attributed to insurance policy holders } \\
\text { Rent }\end{array}$ & NMYO & 54 & 24 & 22 & 19 & 19 & 27 & 25 & 24 & 19 \\
\hline from sectors other than general government & NMYR & 1289 & 1919 & 1901 & 1565 & 1182 & 1229 & 1226 & 1233 & 1162 \\
\hline Total & NMYU & 14226 & 14012 & 11892 & 11742 & 10768 & 10580 & 10901 & 12508 & 12430 \\
\hline Total resources & NMYV & 148427 & 151025 & 154920 & 161774 & 170275 & 173135 & 181702 & 193520 & 190535 \\
\hline \multicolumn{11}{|l|}{ Uses } \\
\hline \multicolumn{11}{|l|}{ Property income, paid } \\
\hline Total interest & NRKB & 30585 & 27911 & 25410 & 26913 & 27013 & 29469 & 30392 & 34232 & .. \\
\hline Total & NMYY & 30585 & 27911 & 25410 & 26913 & 27013 & 29469 & 30392 & 34176 & 36396 \\
\hline After deduction of fixed capital consumption & $-\mathrm{NMXO}$ & -9542 & -9796 & -10289 & -10807 & -11429 & -12174 & -12931 & -14523 & \\
\hline Balance of primary incomes, net & NMZI & 108300 & 113318 & 119221 & 124054 & 131833 & 131492 & 138379 & 145270 & \\
\hline
\end{tabular}

1 See chapter text. 


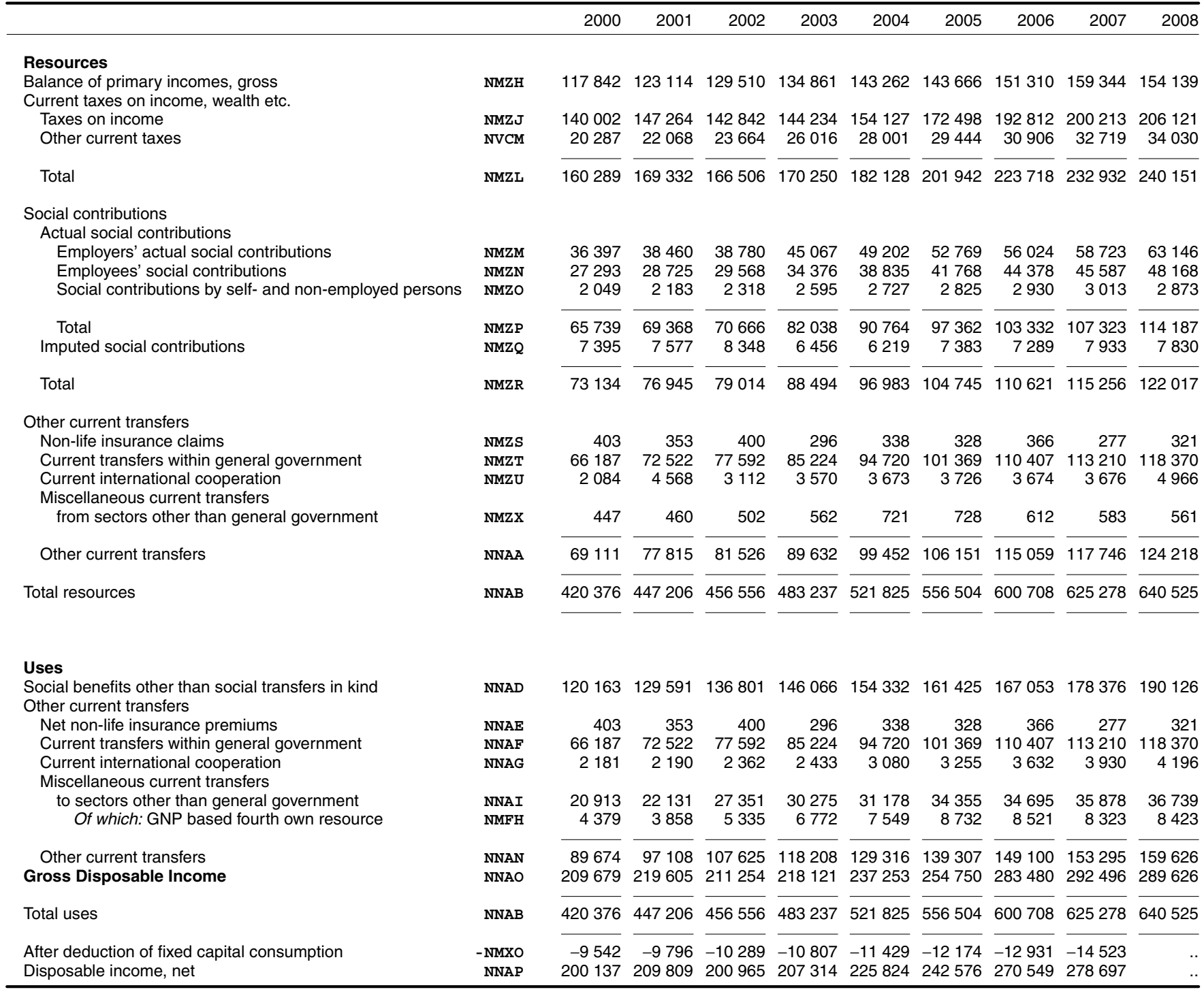

1 See chapter text. 
National accounts

\section{Households and non-profit institutions serving households ${ }^{1}$ \\ Allocation of primary income account \\ United Kingdom. ESA95 sectors S.14 and S.15}

$£$ million

\begin{tabular}{|c|c|c|c|c|c|c|c|c|c|c|}
\hline & & 2000 & 2001 & 2002 & 2003 & 2004 & 2005 & 2006 & 2007 & 2008 \\
\hline \multicolumn{11}{|l|}{ Resources } \\
\hline Operating surplus, gross & QWLS & 49172 & 53000 & 55647 & 60984 & 65182 & 68632 & 71963 & 79843 & 76392 \\
\hline Mixed income, gross & QWLT & 56931 & 61282 & 64967 & 68324 & 72816 & 74858 & 78908 & 83614 & 85673 \\
\hline \multicolumn{11}{|l|}{ Compensation of employees } \\
\hline Wages and salaries & QWLW & 462505 & 491044 & 508681 & 527689 & 548899 & 573932 & 599461 & 627978 & 651550 \\
\hline Employers' social contributions & QWLX & 69824 & 73216 & 78782 & 89263 & 98706 & 107663 & 115290 & 116762 & 118361 \\
\hline Total & QWLY & 532329 & 564260 & 587463 & 616952 & 647605 & 681595 & 714751 & 744740 & 769911 \\
\hline \multicolumn{11}{|l|}{ Property income } \\
\hline Interest & QWLZ & 35488 & 31957 & 26658 & 27251 & 34550 & 40007 & 42706 & 53595 & 46144 \\
\hline Distributed income of corporations & QWMA & 43755 & 49894 & 43787 & 45248 & 45862 & 50704 & 47957 & 44429 & 44292 \\
\hline Attributed property income of insurance policy holders & QWMC & 53081 & 53277 & 52104 & 55008 & 54623 & 64028 & 66640 & 71872 & 75195 \\
\hline Rent & QWMD & 105 & 105 & 106 & 108 & 110 & 110 & 112 & 110 & 115 \\
\hline Total & QWME & 132429 & 135233 & 122655 & 127615 & 135145 & 154849 & 157415 & 170006 & 165746 \\
\hline Total resources & QWMF & 770861 & 813775 & 830732 & 873875 & 920748 & 979934 & 1023037 & 1078203 & 1097722 \\
\hline \multicolumn{11}{|l|}{$\begin{array}{l}\text { Uses } \\
\text { Property income }\end{array}$} \\
\hline Interest & QWMG & 36968 & 33752 & 30512 & 32001 & 43207 & 46739 & 49046 & 67339 & 55876 \\
\hline Rent & QWMH & 215 & 215 & 216 & 220 & 224 & 224 & 228 & 234 & 239 \\
\hline Total & QWMI & 37183 & 33967 & 30728 & 32221 & 43431 & 46963 & 49274 & 67573 & 56115 \\
\hline Balance of primary incomes, gross & QWMU & 733678 & 779808 & 800004 & 841654 & 877317 & 932971 & 973763 & 1010630 & 1041607 \\
\hline Total uses & QWMF & 770861 & 813775 & 830732 & 873875 & 920748 & 979934 & 1023037 & 1078203 & 1097722 \\
\hline \multicolumn{11}{|l|}{ After deduction of } \\
\hline fixed capital consumption & -QWLL & -30518 & -32908 & -36043 & -36903 & -42561 & -43387 & -48789 & -52077 & \\
\hline Balance of primary incomes, net & QWMK & 703160 & 746900 & 763961 & 804751 & 834808 & 889714 & 925140 & 957795 & \\
\hline
\end{tabular}

1 See chapter text.

Source: Office for National Statistics: 02070142131 


\section{Households and non-profit institutions serving households ${ }^{1}$ \\ Secondary distribution of income account}

United Kingdom. ESA95 sectors S.14 and S.15

\begin{tabular}{|c|c|c|c|c|c|c|c|c|c|c|}
\hline & & 2000 & 2001 & 2002 & 2003 & 2004 & 2005 & 2006 & 2007 & 2008 \\
\hline \multicolumn{11}{|l|}{ Resources } \\
\hline Balance of primary incomes, gross & QWMJ & 733678 & 779808 & 800004 & 841654 & 877317 & 932971 & 973763 & 1010630 & 1041607 \\
\hline Imputed social contributions & RVFH & 476 & 502 & 530 & 505 & 499 & 506 & 514 & 518 & 524 \\
\hline Social benefits other than social transfers in kind & QWML & 162833 & 171814 & 182673 & 193596 & 198680 & 211686 & 225891 & 226684 & 247057 \\
\hline \multicolumn{11}{|l|}{ Other current transfers } \\
\hline Non-life insurance claims & QWMM & 15713 & 11723 & 17327 & 13890 & 17479 & 17199 & 20346 & 14842 & 17254 \\
\hline Miscellaneous current transfers & QWMN & 27520 & 29080 & 33041 & 34687 & 34845 & 37840 & 38708 & 39618 & 40094 \\
\hline Total resources & QWMP & 940220 & 992927 & 1033575 & 1084332 & 1128820 & 1200202 & 1259222 & 1292292 & 1346536 \\
\hline \multicolumn{11}{|l|}{ Uses } \\
\hline \multicolumn{11}{|l|}{ Current taxes on income, wealth etc } \\
\hline Taxes on income & QWMQ & 105299 & 111888 & 112171 & 113087 & 119591 & 130200 & 139969 & 151913 & 154039 \\
\hline Other current taxes & NVCO & 19427 & 21166 & 22788 & 25174 & 27077 & 28422 & 29831 & 31608 & 32883 \\
\hline \multicolumn{11}{|l|}{ Social contributions } \\
\hline \multicolumn{11}{|l|}{ Actual social contributions } \\
\hline Employers' actual social contributions & QWMT & 57288 & 60296 & 64805 & 77571 & 87675 & 95732 & 103551 & 104289 & 105339 \\
\hline Employees' social contributions & QWMU & 58807 & 60599 & 62458 & 66490 & 70451 & 78540 & 84129 & 84907 & 90833 \\
\hline Social contributions by self and non-employed & QWMV & 2049 & 2183 & 2318 & 2595 & 2727 & 2825 & 2930 & 3013 & 2873 \\
\hline Total & QWMW & 118144 & 123078 & 129581 & 146656 & 160853 & 177097 & 190610 & 195798 & \\
\hline Imputed social contributions & QWMX & 12536 & 12920 & 13977 & 11692 & 11031 & 11931 & 11739 & 12473 & 13022 \\
\hline Total & QWMY & 130680 & 135998 & 143558 & 158348 & 171884 & 189028 & 202349 & 204682 & 212067 \\
\hline Social benefits other than social transfers in kind & QWMZ & 948 & 977 & 1006 & 987 & 988 & 1000 & 1010 & 1014 & 1020 \\
\hline \multicolumn{11}{|l|}{ Other current transfers } \\
\hline Net non-life insurance premiums & QWNA & 15713 & 11723 & 17327 & 13890 & 17479 & 17199 & 20346 & 14842 & 17254 \\
\hline Miscellaneous current transfers & QWNB & 10865 & 11081 & 11458 & 11930 & 12462 & 13442 & 13274 & 13532 & 13826 \\
\hline Total & QWNC & 26578 & 22804 & 28785 & 25820 & 29941 & 30641 & 33620 & 28374 & 31080 \\
\hline Gross Disposable Income ${ }^{2}$ & QWND & 657288 & 700094 & 725267 & 760916 & 779339 & 820911 & 852443 & 874701 & 915447 \\
\hline After deduction of fixed capital consumption & -QWLL & -30518 & -32908 & -36043 & -36903 & -42561 & -43387 & -48789 & -52077 & .. \\
\hline Disposable income, net & QWNE & 626770 & 667186 & 689224 & 724013 & 736830 & 777654 & 803820 & 819368 & \\
\hline
\end{tabular}

1 See chapter text.

2 Gross household disposable income revalued by the implied households

and NPISH's final consumption expenditure deflator. For more details see table 6.1.4 on page 217 in United Kingdom National Accounts (the Blue book).

\section{Households and non-profit institutions serving households ${ }^{1}$ Use of disposable income account United Kingdom. ESA95 sectors S.14 and S.15}

$£$ million and percentages

\begin{tabular}{|c|c|c|c|c|c|c|c|c|c|c|}
\hline & & 2000 & 2001 & 2002 & 2003 & 2004 & 2005 & 2006 & 2007 & 2008 \\
\hline \multicolumn{11}{|l|}{ Resources } \\
\hline \multirow{2}{*}{$\begin{array}{l}\text { Disposable income, gross } \\
\text { Adjustment for the change in net equity } \\
\text { of households in pension funds }\end{array}$} & QWND & 657288 & 700094 & 725267 & 760916 & 779339 & 820911 & 852443 & 874701 & 915447 \\
\hline & NSSE & 14154 & 16038 & 17784 & 21377 & 29468 & 32888 & 31714 & 39816 & \\
\hline Total resources & NSSF & 671442 & 716132 & 743051 & 782293 & 808807 & 853799 & 884157 & 914517 & 947103 \\
\hline Individual consumption expenditure & NSSG & 640089 & 672889 & 707386 & 742276 & 776250 & 810667 & 846868 & 894389 & 928552 \\
\hline Saving, gross & NSSH & 31353 & 43243 & 35665 & 40017 & 32557 & 43132 & 37289 & 20128 & 18551 \\
\hline Total uses & NSSF & 671442 & 716132 & 743051 & 782293 & 808807 & 853799 & 884157 & 914517 & 947103 \\
\hline Saving ratio (percentages) & RVGL & 4.7 & 6.0 & 4.8 & 5.1 & 4.0 & 5.1 & 4.2 & 2.2 & 2.0 \\
\hline
\end{tabular}


National accounts

\section{The sector accounts: key economic indicators ${ }^{1}$ \\ United Kingdom}

$£$ million and indices $(2003=100)$

\begin{tabular}{|c|c|c|c|c|c|c|c|c|c|c|}
\hline & & 2000 & 2001 & 2002 & 2003 & 2004 & 2005 & 2006 & 2007 & 2008 \\
\hline \multicolumn{11}{|l|}{ Net lending/borrowing by: } \\
\hline Non-financial corporations & EABO & -4614 & -6366 & 10549 & 22971 & 30983 & 22285 & 27728 & 36780 & 41908 \\
\hline Financial corporations & NHCQ & -26988 & -22123 & 4386 & 13451 & 18477 & 5036 & -3275 & 21964 & 56010 \\
\hline General government & NNBK & 13168 & 6546 & -20389 & -37206 & -39959 & -41239 & -34739 & -37107 & -75807 \\
\hline Households and NPISH's & NSSZ & -6436 & 1044 & -12477 & -12091 & -29533 & -17731 & -33643 & -56382 & -40715 \\
\hline Rest of the world & NHRB & 24084 & 19784 & 17725 & 16841 & 23136 & 31188 & 44056 & 37761 & 20988 \\
\hline \multicolumn{11}{|l|}{ Gross trading profits } \\
\hline Continental shelf profits & CAGJ & 21458 & 20397 & 18742 &.. & .. & . & & & \\
\hline Others & CAED & 152682 & 151364 & 160068 & 173584 & 182976 & 185873 & 195399 & 217755 & 214863 \\
\hline Rental of buildings & FCBW & 11747 & 12394 & 12904 & 13891 & 16097 & 16936 & 17350 & 18011 & 18930 \\
\hline less Holding gains of inventories & $-D L Q Z$ & -2941 & 438 & -2856 & -4266 & -2786 & -4058 & -3444 & -6644 & -9323 \\
\hline Gross operating surplus & NRJK & 182115 & 183157 & 188444 & 201091 & 214851 & 222175 & 236916 & 254156 & 259884 \\
\hline Household gross disposable income & QWND & 657288 & 700094 & 725267 & 760916 & 779339 & 820911 & 852443 & 874701 & 915447 \\
\hline Implied deflator of household and NPISH individual & & & & & & & & & & \\
\hline \multicolumn{11}{|l|}{ Real household disposable income: } \\
\hline Chained volume measures (Reference year 2003) & RVGK & 693322 & 724114 & 738900 & 760916 & 767096 & 788338 & 799898 & 801135 & 818624 \\
\hline Indices $(2003=100)$ & OSXR & 91.1 & 95.2 & 97.1 & 100.0 & 100.8 & 103.6 & 105.1 & 105.3 & 107.6 \\
\hline Gross saving & NSSH & 31353 & 43243 & 35665 & 40017 & 32557 & 43132 & 37289 & 20128 & 18551 \\
\hline Households total resources & NSSU & 780739 & 834590 & 873867 & 926247 & 957751 & 1014255 & 1057272 & 1097319 & 1140361 \\
\hline Saving ratio (percentages) & RVGL & 4.7 & 6.0 & 4.8 & 5.1 & 4.0 & 5.1 & 4.2 & 2.2 & 2.0 \\
\hline
\end{tabular}

1 See chapter text.

Source: Office for National Statistics: 02070142083 


\begin{tabular}{|c|c|c|c|c|c|c|c|c|c|c|}
\hline & & 2000 & 2001 & 2002 & 2003 & 2004 & 2005 & 2006 & 2007 & 2008 \\
\hline \multicolumn{11}{|l|}{ Durable goods } \\
\hline \multicolumn{11}{|l|}{ Furnishings, household equipment and } \\
\hline routine maintenance of the house & LLIJ & 18006 & 19275 & 20470 & 21595 & 22316 & 22976 & 23622 & 24960 & 25079 \\
\hline Health & LLIK & 1997 & 2109 & 2411 & 2604 & 2467 & 2368 & 2650 & 2713 & 2710 \\
\hline Transport & LLIL & 33291 & 35864 & 36574 & 38016 & 38643 & 38361 & 39047 & 40886 & 37707 \\
\hline Communication & LLIM & 601 & 636 & 644 & 810 & 850 & 900 & 900 & 927 & 1005 \\
\hline Recreation and culture & LLIN & 14878 & 15970 & 16471 & 17752 & 19058 & 20180 & 21012 & 21441 & 21702 \\
\hline Miscellaneous goods and services & LLIO & 3403 & 3750 & 4204 & 4284 & 4739 & 4636 & 5283 & 5507 & 5754 \\
\hline Total durable goods & UTIA & 72176 & 77604 & 80774 & 85061 & 88073 & 89421 & 92514 & 96434 & 93957 \\
\hline \multicolumn{11}{|l|}{ Semi-durable goods } \\
\hline $\begin{array}{l}\text { Clothing and footwear } \\
\text { Furnishings, household equipment and }\end{array}$ & LLJL & 34759 & 36092 & 38351 & 40389 & 42114 & 42999 & 44178 & 45841 & 46587 \\
\hline routine maintenance of the house & LLJM & 11677 & 12400 & 13361 & 13932 & 13502 & 13396 & 13987 & 13788 & 13294 \\
\hline Transport & LLJN & 2772 & 2783 & 3112 & 3423 & 3048 & 3444 & 3438 & 3567 & 4249 \\
\hline Recreation and culture & LLJO & 20405 & 21606 & 23910 & 26009 & 26544 & 26659 & 26671 & 26985 & 27318 \\
\hline Miscellaneous goods and services & LLJP & 2018 & 2427 & 2886 & 3356 & 3477 & 3278 & 3448 & 3658 & 3382 \\
\hline Total semi-durable goods & UTIQ & 71631 & 75308 & 81620 & 87109 & 88685 & 89776 & 91722 & 93839 & 94830 \\
\hline \multicolumn{11}{|l|}{ Non-durable goods } \\
\hline Food \& drink & $\mathrm{ABZV}$ & 58628 & 59804 & 61310 & 63174 & 64830 & 67187 & 69410 & 77144 & 84993 \\
\hline Alcohol \& tobacco & $A D F L$ & 24617 & 25158 & 25966 & 27297 & 28101 & 28437 & 30061 & 30947 & 31120 \\
\hline Housing, water, electricity, gas and & & & & & & & & & & \\
\hline other fuels & LLIX & 22265 & 23076 & 23444 & 24241 & 27439 & 29375 & 33936 & 35824 & 41582 \\
\hline Furnishings, household equipment and & & & & & & & & & & \\
\hline routine maintenance of the house & LLIY & 2786 & 2972 & 3169 & 3338 & 3879 & 3873 & 4091 & 4095 & 4029 \\
\hline Health & LLIZ & 3268 & 3613 & 3855 & 3938 & 4457 & 4525 & 4491 & 4601 & 4859 \\
\hline Transport & LLJA & 19987 & 19391 & 19129 & 20072 & 21161 & 24183 & 25089 & 26359 & 29202 \\
\hline Recreation and culture & LLJB & 12959 & 13107 & 13392 & 13507 & 13976 & 14359 & 14879 & 15305 & 15660 \\
\hline Miscellaneous goods and services & LLJC & 9463 & 9884 & 11272 & 12602 & 13187 & 13276 & 14208 & 15579 & 16270 \\
\hline Total non-durable goods & UTII & 153973 & 157005 & 161537 & 168169 & 177030 & 185215 & 196165 & 209854 & 227715 \\
\hline Total goods & UTIE & 297780 & 309917 & 323931 & 340339 & 353788 & 364412 & 380401 & 400127 & 416502 \\
\hline \multicolumn{11}{|l|}{ Services } \\
\hline Clothing and footwear & LLJD & 720 & 730 & 741 & 766 & 682 & 760 & 824 & 847 & 920 \\
\hline $\begin{array}{l}\text { other fuels } \\
\text {. }\end{array}$ & LLJE & 85785 & 92829 & 97794 & 104810 & 110605 & 117947 & 125760 & 133758 & 138727 \\
\hline Furnishings, household equipment and & & & & & & & & & & \\
\hline routine maintenance of the house & LLJF & 3206 & 3327 & 3448 & 3601 & 3772 & 4084 & 3791 & 3685 & 3904 \\
\hline Health & LLJG & 3943 & 4254 & 4512 & 4793 & 5105 & 5413 & 5603 & 6184 & 6090 \\
\hline Transport & LLJH & 37002 & 38397 & 41332 & 43058 & 45604 & 48180 & 50923 & 52912 & 55678 \\
\hline Communication & LLJI & 12755 & 13521 & 14031 & 14844 & 15944 & 16308 & 16532 & 17089 & 17261 \\
\hline Recreation and culture & LLJJ & 21912 & 22769 & 25349 & 27118 & 29263 & 31218 & 32528 & 34736 & 35588 \\
\hline Education & ADIE & 9534 & 9409 & 9381 & 9610 & 11094 & 11762 & 12432 & 13703 & 14100 \\
\hline Restaurants and hotels & $\mathrm{ADIF}$ & 68557 & 71620 & 76426 & 78902 & 82476 & 84808 & 86729 & 90549 & 93248 \\
\hline Miscellaneous goods and services & LLJK & 68423 & 71481 & 73456 & 74609 & 77229 & 83414 & 87259 & 93679 & 95142 \\
\hline Total services & UTIM & 311837 & 328337 & 346470 & 362111 & 381774 & 403894 & 422381 & 447142 & 460658 \\
\hline \multicolumn{11}{|l|}{$\begin{array}{l}\text { Final consumption expenditure in the UK } \\
\text { by resident and non-resident households } \\
\text { (domestic concept) }\end{array}$} \\
\hline Final consumption expenditure outside the UK & & & & & & & & & & \\
\hline by UK resident households & ABTA & 21654 & 22907 & 24435 & 26314 & 27550 & 29028 & 30389 & 31702 & 33325 \\
\hline less Final consumption expenditure in the UK & & & & & & & & & & \\
\hline by households resident in the rest of the world & CDFD & -14713 & -13383 & -13872 & -14156 & -15610 & -17069 & -18512 & -18991 & -19483 \\
\hline $\begin{array}{l}\text { Final consumption expenditure by UK resident } \\
\text { households in the UK and abroad }\end{array}$ & & & & & & & & & & \\
\hline (national concept) & $\mathrm{ABPB}$ & 616558 & 647778 & 680964 & 714608 & 747502 & 780265 & 814659 & 859980 & 891002 \\
\hline
\end{tabular}




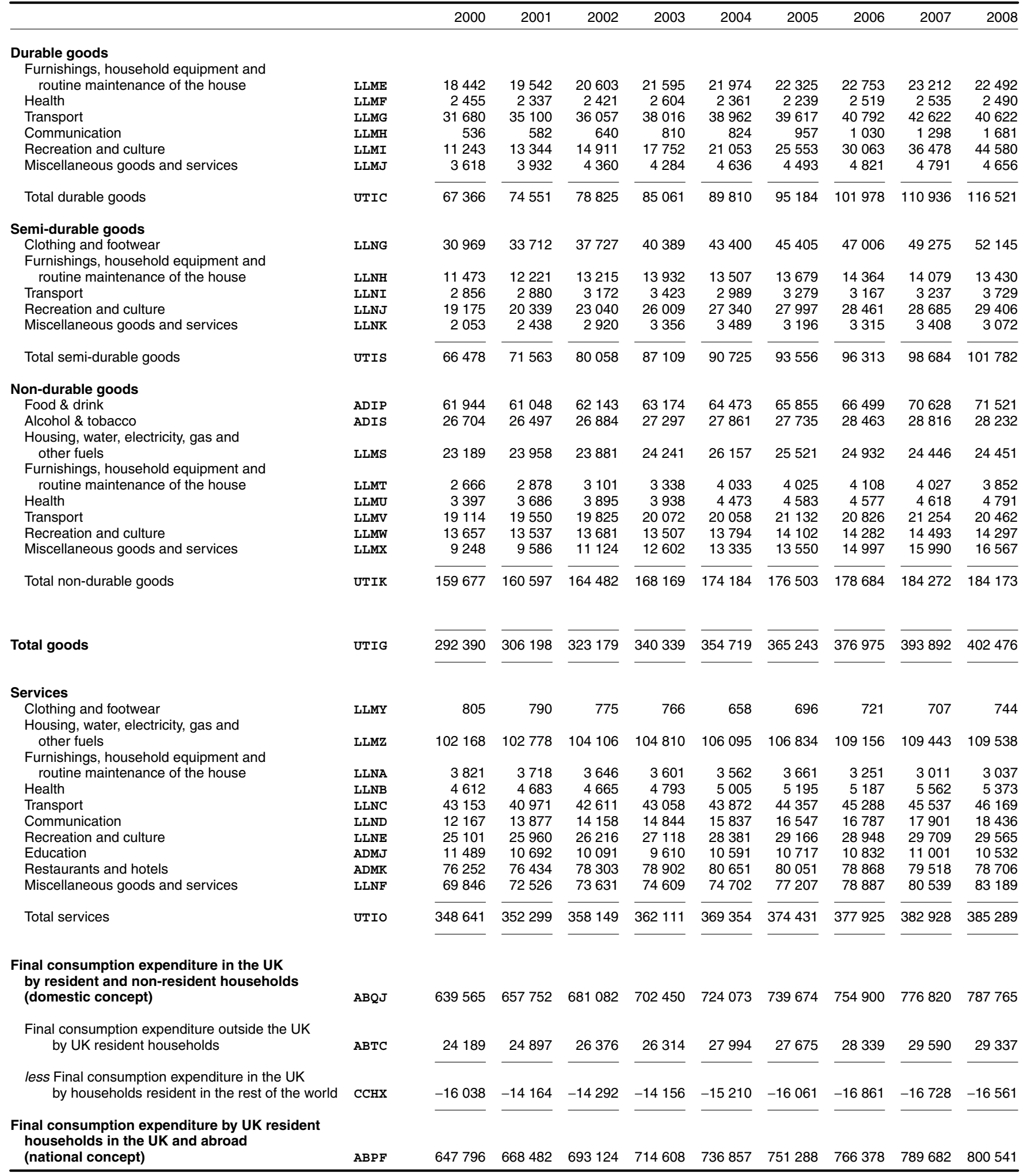

1 See chapter text. Additional detail is published in Consumer Trends and Source: Office for National Statistics: 02070142116 


\section{FINAL CONSUMPTION EXPENDITURE OF HOUSEHOLDS}

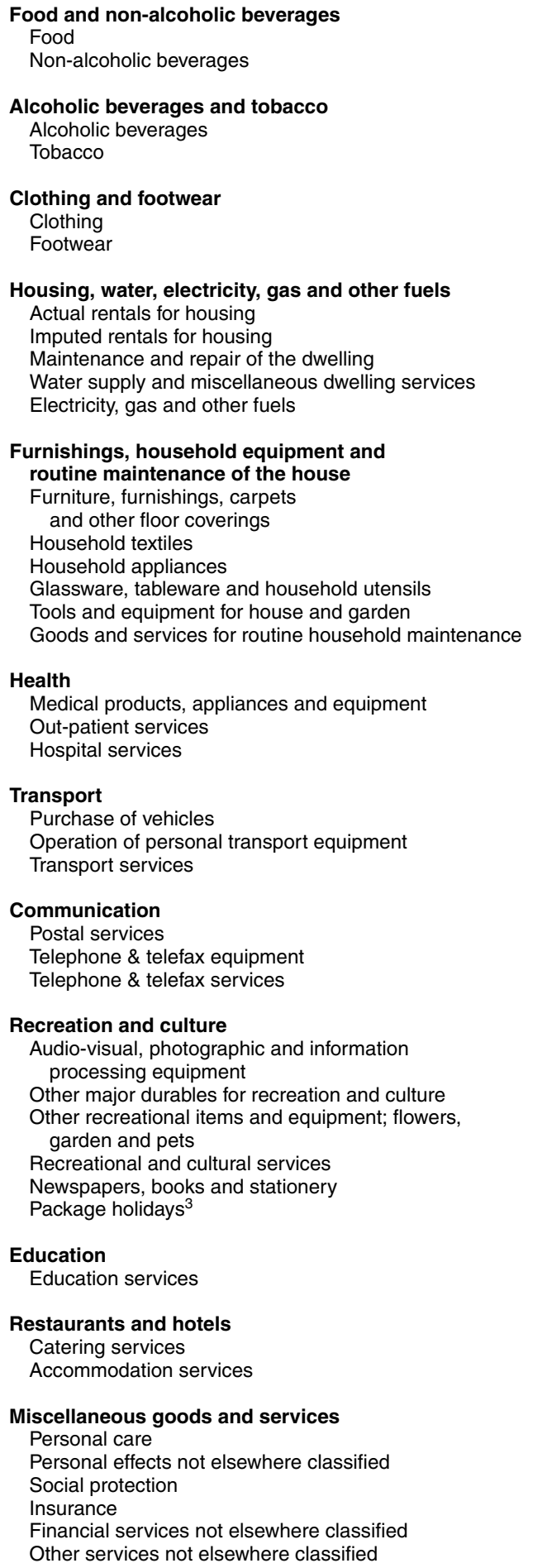

Final consumption expenditure in the UK by resident and non-resident households (domestic concept)

Final consumption expenditure outside the UK by UK resident households

less Final consumption expenditure in the UK by households resident in the rest of the world

Final consumption expenditure by UK resident households in the UK and abroad (national concept)

\begin{tabular}{l} 
ABZ \\
ABZ \\
ADF \\
ADF \\
ADF \\
ADF \\
ADF \\
ADF \\
ADF \\
ADF \\
ADF \\
ADF \\
ADF \\
ADF \\
ADF \\
ADI \\
ADI \\
ADI \\
ADI \\
ADI \\
ADI \\
ADF \\
ADI \\
ADI \\
ADG \\
ADI \\
ADI \\
ADI \\
ADG \\
ADG \\
ADG \\
ADG \\
ADG \\
ADG \\
ADG \\
ADG \\
ADG \\
ADG \\
ADG \\
ADG \\
ADG \\
ADE \\
\hline
\end{tabular}

ABZV

ABW

ADFM

ADFN

ADFP

ADFS

ADFT

ADFU

ADFV

ADFX

ADFY

ADFZ

ADGG

ADGL

ADGM

ADGO

ADGP

$A D G Q$

ADGR

ADGT

ADGU

ADGX

CDEF

ADWP

ADGY

ADGZ

ADHL

$\mathrm{ADHZ}$

ADIE

ADIF

ADIG

ADII

ADIJ

ADIK

ADIM

ADIO $\begin{array}{llll}59804 & 61310 & 63174\end{array}$

$\begin{array}{rrrr}6723 & 7062 & 7326 & 7667\end{array}$

$24617 \quad 25158$

$25966 \quad 27297$

$39092 \quad 41155$

$33927 \quad 35689$

8050115905

$\begin{array}{rrrr}23595 & 25302 & 25828 & 27610\end{array}$

$\begin{array}{llll}54378 & 59581 & 63279 & 68458\end{array}$

$10512 \quad 11340$

$5033 \quad 5059$

$14532 \quad 14623$

$\begin{array}{ll}63279 & 68458 \\ 12306 & 12615\end{array}$

$\begin{array}{rr}12306 & 12615 \\ 5222 & 5438\end{array}$

14603

14930

$35675 \quad 37974$

$13758 \quad 14362$

$4465 \quad 4636$

$5156 \quad 5758$

$4231 \quad 4609$

$\begin{array}{ll}2722 & 2977 \\ 5343 & 5632\end{array}$

40448

42466

$15591 \quad 16789$

$5086 \quad 5452$

$5715 \quad 5578$

$4710 \quad 4701$

$\begin{array}{ll}3355 & 3589 \\ 5991 & 6357\end{array}$

6357

$9208 \quad 9976$

$5265 \quad 5722$

$2178 \quad 2344$

$\begin{array}{rr}10778 & 11335 \\ 6266 & 6542\end{array}$

$2422 \quad 2553$

$2090 \quad 2240$

9305

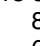

601

11882

70154

17034

3944

18636

20272

10268

23543

6046

14157

870
636

14675

675
878
644

15654

654
890
810

12651

13153

13954

79122

84386

805

17580

467

19408

126

$22475 \quad 23894$

$23555 \quad 25278$

$10369 \quad 10680$
$14222 \quad 14458$

$38816 \quad 40507$

64830

8163

$28101 \quad 28437$

$13035 \quad 13065$

$15066 \quad 15372$

$42796 \quad 43759$

37609

6150

$138044 \quad 14732$

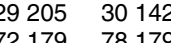

$13056 \quad 13538$

$5831 \quad 6279$

$16873 \quad 19184$

69410

60627
8783

77144

84993

10203

$\begin{array}{lll}30947 & 31120\end{array}$

$\begin{array}{lll}13924 & 14927 & 15024\end{array}$

$16137 \quad 16020 \quad 16096$

$45002 \quad 46688 \quad 47507$

$38419 \quad 39765 \quad 40433$

$\begin{array}{lll}6583 & 6923 \quad 7074\end{array}$

$169586 \quad 180309$

$32716 \quad 34648 \quad 35205$

$82388 \quad 87729 \quad 92007$

$\begin{array}{lll}13287 & 14233 & 14124\end{array}$

$\begin{array}{rrr}6820 & 7228 & 7760\end{array}$

$4485 \quad 25744 \quad 31213$

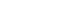

$9534 \quad 9409$

9381

9610

$43469 \quad 44329$

45491

46528

46306

$17168 \quad 17309$

6028

3870

4006
7098

6391

4210

4090

17702

5146
6704

4122

4445

18813

5423

6729
3494

3494
4739
7330

7330

12029

6924

2747
2358

12306

6893

12744

13498

$\begin{array}{rr}7141 & 7314 \\ 2983 & 3460\end{array}$

3460
2724

2504

2620

18597

5094

6698

3495

7587

$\begin{array}{llllllllll}68557 & 71620 & 76426 & 78902 & 82476 & 84808 & 86729 & 90549 & 93248\end{array}$ $\begin{array}{lllllllll}59019 & 62449 & 66701 & 68839 & 72399 & 74294 & 75501 & 78249 & 80094\end{array}$

$\begin{array}{lllllllll}9538 & 9171 & 9725 & 10063 & 10077 & 10514 & 11228 & 12300 & 13154\end{array}$

$\begin{array}{llllllllll}83307 & 87542 & 91818 & 94851 & 98632 & 104604 & 110198 & 118423 & 120548\end{array}$

$\begin{array}{llllllrrr}13883 & 14626 & 16444 & 18181 & 19538 & 20022 & 21020 & 22623 & 22990\end{array}$

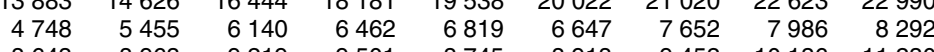

$\begin{array}{lllllllll}8643 & 8963 & 9219 & 9501 & 8745 & 8918 & 9452 & 10136 & 11230\end{array}$

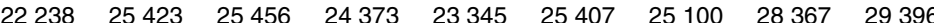

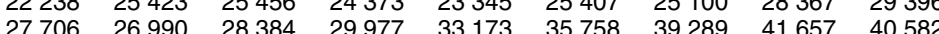

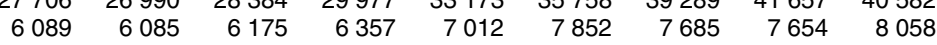

$609617 \quad 638254 \quad 670401 \quad 702450 \quad 735562 \quad 768306 \quad 802782 \quad 847269 \quad 877160$

ABTA

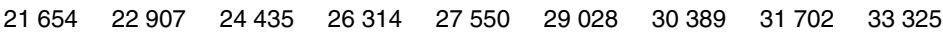

CDFD

\begin{tabular}{llllllllll}
-14713 & -13383 & -13872 & -14 & 156 & -15610 & -17069 & -18512 & -18991 & -19 \\
\hline
\end{tabular} 
National accounts

$\begin{array}{lllllll}2000 & 2001 & 2002 & 2003 & 2004 & 2005 & 2006\end{array}$

FINAL CONSUMPTION EXPENDITURE OF UK RESIDENT HOUSEHOLDS

Final consumption expenditure of UK resident households in the UK and abroad

FINAL INDIVIDUAL CONSUMPTION EXPENDITURE OF NPISH

Final individual consumption expenditure of NPISH

ABNV

23531

25111

26422

27668

28748

30402

32209

34409

37550

FINAL INDIVIDUAL CONSUMPTION EXPENDITURE OF OF GENERAL GOVERNMENT

Health

Recreation and culture

Education

Social protection

Housing

QYот $\quad \begin{array}{llllll}53236 & 58032 & 63388 & 69888 & 76855\end{array}$

$\begin{array}{lrrrrr}\text { QYSU } & 3898 & 4049 & 4335 & 4513 & 4272\end{array}$

$\begin{array}{llllll}\text { QYSE } & 31521 & 33900 & 37535 & 39876 & 42727\end{array}$

$\begin{array}{lllllll}\text { OYSP } & 18055 & 19441 & 22464 & 25517 & 28028\end{array}$

83579

11058

85331

47235

Final individual consumption expenditure of general government

NNAQ

109297

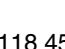

$-$

NQEO

$749386791347838202886230925194971123 \quad 1019983 \quad 1077191 \quad 1121810$ actual individual consumption

1 See chapter text.

Source: Office for National Statistics: 02070142116

2 "Purpose" or "function" classifications are designed to indicate the "soci-

economic objectives" that institutional units aim to achieve through various

kinds of outlays. COICOP is the Classification of Individual Consumption by

Purpose and applies to households. COPNI is the Classification of the Pur-

poses of Non-Profit Institutions Serving Households and COFOG the Clas-

sification of the Functions of Government. The introduction of ESA95 coin-

cides with the redefinition of these classifications and data will be available

on a consistent basis for all European Union member states.

3 Package holidays data are dispersed between components (transport etc). 


\section{FINAL CONSUMPTION EXPENDITURE OF HOUSEHOLDS}

Food and non-alcoholic beverages

Food

Non-alcoholic beverages

Alcoholic beverages and tobacco

Alcoholic beverages

Tobacco

Clothing and footwear

Clothing

Footwear

Housing, water, electricity, gas and other fuels

Actual rentals for housing

Imputed rentals for housing

Maintenance and repair of the dwelling

Water supply and miscellaneous dwelling services

Electricity, gas and other fuels

Furnishings, household equipment and

routine maintenance of the house

Furniture, furnishings, carpets

and other floor covering

Household textiles

Household appliances

Glassware, tableware and household utensils

Tools and equipment for house and garden

Goods and services for routine household maintenance

Health

Medical products, appliances and equipment

Out-patient services

Hospital services

Transport

Purchase of vehicles

Operation of personal transport equipment

Transport services

Communication

Postal services

Telephone \& telefax equipment

Telephone \& telefax services

Recreation and culture

Audio-visual, photographic and information processing equipment

Other major durables for recreation and culture

Other recreational items and equipment; flowers, gardens and pets

Recreational and cultural services

Newspapers, books and stationery

Package holidays ${ }^{3}$

\section{Education}

Education services

Restaurants and Hotels

Catering services

Accommodation services

Miscellaneous goods and services

Personal care

Personal effects not elsewhere classified

Social protection

Insurance

Financial services not elsewhere classified

Other services not elsewhere classified

Final consumption expenditure in the UK by resident and non-resident households (domestic concept)

Final consumption expenditure outside the UK by UK resident households

less Final consumption expenditure in the UK by households resident in the rest of the world

Final consumption expenditure by UK resident households in the UK and abroad (national concept)
ADIR

ADIS

ADIT

ADIU

ADIW

ADIX

ADIY

ADIZ

ADJA

ADJB

ADJC

ADJD

ADJE

ADJF

ADJG

ADJH

ADJI

ADJU

ADJK

ADJL

ADJM

ADJN

ADJO

ADJP

ADJQ

ADJR

ADJS

ADJT

ADJU

CCGZ

$A D Q F$

ADOG

ADJV

ADJW

ADJX

ADJY

ADJZ

$A D K M$

ADMI

ADMJ

ADMK

ADML

ADMM

ADMN

ADMO

ADMP

ADMO

ADMR

ADMS

ADMT

ABQJ

$639565 \quad 657752 \quad 681082 \quad 702450 \quad 724073 \quad 739674 \quad 754900 \quad 776820 \quad 787765$

ABTC

CCHX

$\begin{array}{lllllllll}61944 & 61048 & 62143 & 63174 & 64473 & 65855 & 66499 & 70628 & 71521\end{array}$

$\begin{array}{lllllllll}55255 & 53992 & 54835 & 55507 & 56240 & 57305 & 57969 & 61282 & 62255\end{array}$

$\begin{array}{llllrrrrr}6725 & 7063 & 7312 & 7667 & 8233 & 8550 & 8530 & 9346 & 9266\end{array}$

$\begin{array}{lllllllll}26704 & 26497 & 26884 & 27297 & 27861 & 27735 & 28463 & 28816 & 28232\end{array}$

$\begin{array}{lllllllll}10476 & 10831 & 11516 & 12027 & 13204 & 13331 & 14005 & 15056 & 14888\end{array}$

$\begin{array}{lllllllll}16341 & 15716 & 15380 & 15270 & 14657 & 14404 & 14458 & 13760 & 13344\end{array}$

$\begin{array}{llllllllll}31744 & 34485 & 38499 & 41155 & 44058 & 46101 & 47727 & 49982 & 52889\end{array}$

$\begin{array}{lllllllll}27394 & 29827 & 33315 & 35689 & 37924 & 39688 & 40823 & 42816 & 45531\end{array}$

$\begin{array}{lllllllll}4360 & 4660 & 5185 & 5466 & 6134 & 6413 & 6904 & 7166 & 7358\end{array}$

$\begin{array}{llllllllll}125299 & 126749 & 127979 & 129051 & 132252 & 132355 & 134088 & 133889 & 133989\end{array}$

$\begin{array}{llllllllll}27345 & 27418 & 27084 & 27610 & 27933 & 27644 & 29071 & 28877 & 28721\end{array}$

$\begin{array}{llllllllll}65704 & 66495 & 67872 & 68458 & 69438 & 70672 & 71154 & 71518 & 72147\end{array}$

$\begin{array}{lllllllll}11675 & 12139 & 12702 & 12615 & 13581 & 12793 & 12115 & 12400 & 11673\end{array}$

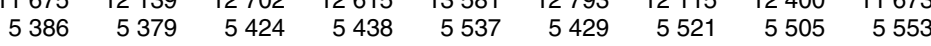

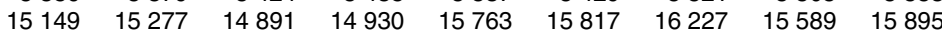

$\begin{array}{lllllllll}36305 & 38310 & 40552 & 42466 & 43076 & 43690 & 44476 & 44329 & 42811\end{array}$

$\begin{array}{lllllllll}14514 & 14860 & 15896 & 16789 & 16751 & 16425 & 16419 & 16795 & 15775\end{array}$

$\begin{array}{lllllllll}4361 & 4534 & 5043 & 5452 & 5202 & 4944 & 5283 & 5618 & 5289\end{array}$

$\begin{array}{lllllllll}4922 & 5549 & 5566 & 5578 & 6059 & 6527 & 7033 & 6886 & 6801\end{array}$

$\begin{array}{lllllllll}4266 & 4655 & 4717 & 4701 & 3866 & 4317 & 4231 & 3554 & 3464\end{array}$

$\begin{array}{lllllllll}2590 & 2856 & 3238 & 3589 & 4136 & 4293 & 4611 & 4837 & 4886\end{array}$

$\begin{array}{lllllllll}5708 & 5859 & 6092 & 6357 & 7062 & 7184 & 6899 & 6639 & 6596\end{array}$

$\begin{array}{lllllllll}10421 & 10697 & 10980 & 11335 & 11839 & 12017 & 12283 & 12715 & 12654\end{array}$

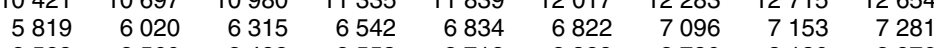

$\begin{array}{lllllllll}2528 & 2560 & 2492 & 2553 & 2712 & 2829 & 2780 & 3130 & 2976\end{array}$

$\begin{array}{lllllllll}2082 & 2122 & 2173 & 2240 & 2293 & 2366 & 2407 & 2432 & 2397\end{array}$

$\begin{array}{lllllllll}96209 & 98485 & 101621 & 104569 & 105881 & 108385 & 110073 & 112650 & 110982\end{array}$

$\begin{array}{lllllllll}31680 & 35100 & 36057 & 38016 & 38962 & 39617 & 40792 & 42622 & 40622\end{array}$

$\begin{array}{llllllllll}39124 & 39225 & 40668 & 40507 & 40508 & 41553 & 40907 & 40790 & 40240\end{array}$

$\begin{array}{lllllllll}25913 & 24214 & 24965 & 26046 & 26411 & 27215 & 28374 & 29238 & 30120\end{array}$

$\begin{array}{lllllllll}12698 & 14452 & 14796 & 15654 & 16661 & 17504 & 17817 & 19199 & 20117\end{array}$

$\begin{array}{rrrrrrrrr}916 & 901 & 906 & 890 & 932 & 1034 & 1120 & 1293 & 1294 \\ 536 & 582 & 640 & 810 & 824 & 957 & 1030 & 1298 & 1681\end{array}$

$\begin{array}{lllllllll}11264 & 12978 & 13254 & 13954 & 14905 & 15513 & 15667 & 16608 & 17142\end{array}$

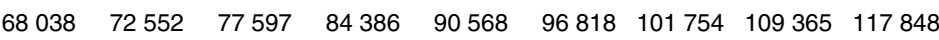

$\begin{array}{lllllllll}13022 & 14690 & 16301 & 19408 & 23041 & 27358 & 30782 & 36317 & 43900\end{array}$

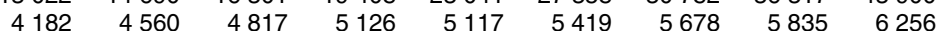

$\begin{array}{lllllllll}17455 & 18980 & 21642 & 23894 & 24842 & 25541 & 26943 & 28519 & 29586\end{array}$

$\begin{array}{llllllllll}23206 & 24049 & 24333 & 25278 & 26522 & 26995 & 26977 & 27537 & 27768\end{array}$

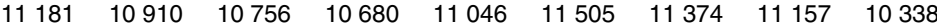

$\begin{array}{lllllllll}11489 & 10692 & 10091 & 9610 & 10591 & 10717 & 10832 & 11001 & 10532\end{array}$

$\begin{array}{lllllllll}76252 & 76434 & 78303 & 78902 & 80651 & 80051 & 78868 & 79518 & 78706\end{array}$

$\begin{array}{lllllllll}65644 & 66815 & 68462 & 68839 & 70766 & 70248 & 68896 & 68974 & 67697\end{array}$

$\begin{array}{lllllllll}10610 & 9620 & 9843 & 10063 & 9885 & 9803 & 9972 & 10544 & 11009\end{array}$

$\begin{array}{llllllllll}84709 & 88415 & 92015 & 94851 & 96162 & 98446 & 102020 & 104728 & 107484\end{array}$

$\begin{array}{llllllrrr}14251 & 14719 & 16526 & 18181 & 19451 & 19747 & 21035 & 22020 & 22099\end{array}$

$\begin{array}{rrrrrrrrr}4922 & 5607 & 6289 & 6462 & 6730 & 6481 & 7134 & 7132 & 6981\end{array}$

$\begin{array}{lllllllll}10357 & 10058 & 9760 & 9501 & 8416 & 8062 & 8013 & 8259 & 8775\end{array}$

$\begin{array}{lllllllll}23526 & 25453 & 24880 & 24373 & 22633 & 23523 & 24125 & 24935 & 25295\end{array}$

$\begin{array}{llllllllll}24666 & 25875 & 28040 & 29977 & 32391 & 33760 & 35338 & 36247 & 38169\end{array}$

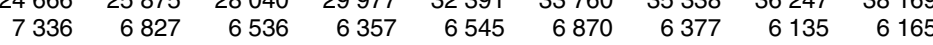

$\mathrm{ABPF}$

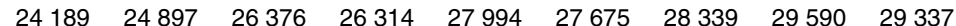

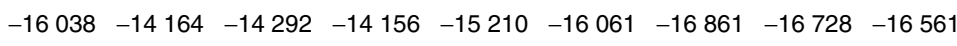

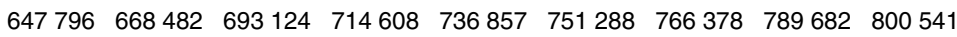


National accounts

$2000 \quad 2001 \quad 2002$

Final consumption expenditure of UK resident households in the UK and abroad

FINAL INDIVIDUAL CONSUMPTION EXPENDITURE OF NPISH

Final individual consumption expenditure of NPISH

FINAL INDIVIDUAL CONSUMPTION EXPENDITURE OF GENERAL GOVERNMENT

Health

Recreation and culture

Education

Social protection

Housing

Final individual consumption expenditure of general government

Total, individual consumption expenditure/ actual individual consumption
ABNU

27536

27567

27576

27668

27198

27212

28289

29485

29802

$\begin{array}{rrrrrr}\text { EMOA } & 58517 & 61019 & 63272 & 65611 & 68758\end{array}$

$\begin{array}{lrrrrrr}\text { QYXK } & 4051 & 3968 & 4470 & 4717 & . \\ \text { EMOB } & 36876 & 37100 & 37535 & 37732 & 37944\end{array}$

QYXM

$23454 \quad 23645 \quad 24864 \quad 25843$

27213

27602

NSZK

$111763 \quad 114159117238 \quad 120288$

YBIO $\quad 806541 \quad 830840 \quad 860237 \quad 886230 \quad 912715 \quad 929549 \quad 947894 \quad 975182$

1 See chapter text.

2 "Purpose" or "function" classifications are designed to indicate the "soci-

economic objectives" that institutional units aim to achieve through various

kinds of outlays. COICOP is the Classification of Individual Consumption by

Purpose and applies to households. COPNI is the Classification of the Pur-

poses of Non-Profit Institutions Serving Households (NPISH) and COFOG

the Classification of the Functions of Government. The introduction of

ESA95 coincides with the redefinition of these classifications and data will

be available on a consistent basis for all European Union member states.

3 Package holidays data are dispersed between components (transport etc).

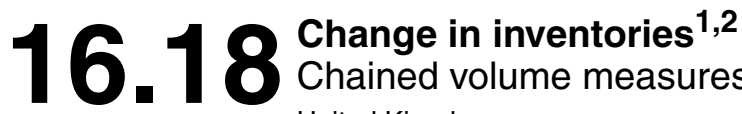 \\ Chained volume measures, reference year 2003 \\ United Kingdom}

Reference year 2003, £ million

\begin{tabular}{|c|c|c|c|c|c|c|c|c|c|c|}
\hline & \multirow{2}{*}{$\begin{array}{r}\text { Mining } \\
\text { and } \\
\text { quarrying }\end{array}$} & \multicolumn{4}{|c|}{ Manufacturing industries } & \multirow{2}{*}{$\begin{array}{r}\text { Electricity, } \\
\text { gas and } \\
\text { water } \\
\text { supply }\end{array}$} & \multicolumn{2}{|c|}{$\begin{array}{l}\text { Distributive } \\
\text { trades }\end{array}$} & \multirow[b]{2}{*}{$\begin{array}{l}\text { Other } \\
\text { industries }^{4}\end{array}$} & \multirow[b]{2}{*}{$\begin{array}{l}\text { Change in } \\
\text { inventories }\end{array}$} \\
\hline & & $\begin{array}{r}\text { Materials } \\
\text { and fuel }\end{array}$ & $\begin{array}{r}\text { Work in } \\
\text { progress }\end{array}$ & $\begin{array}{r}\text { Finished } \\
\text { goods }\end{array}$ & Total & & Wholesale ${ }^{3}$ & Retail $^{3}$ & & \\
\hline & FADO & FBID & FBIE & FBIF & $\mathrm{DHBH}$ & FADP & FAJM & FBYH & DLWV & $A B M Q$ \\
\hline 1999 & -325 & 503 & -259 & -430 & -157 & -167 & 1743 & 1722 & 3464 & 5803 \\
\hline 2000 & -263 & 543 & 358 & 418 & 1318 & 202 & 1939 & 1480 & -283 & 4648 \\
\hline 2001 & 87 & -513 & 369 & 160 & 17 & 16 & 887 & 1113 & 3458 & 5577 \\
\hline 2002 & -37 & -496 & -149 & -372 & -1017 & -132 & 788 & 1716 & 971 & 2289 \\
\hline 2003 & -66 & -198 & -650 & -138 & -986 & -13 & 407 & 1241 & 3399 & 3982 \\
\hline 2004 & -47 & 33 & -592 & -278 & -837 & 8 & 304 & 1000 & 3943 & 4371 \\
\hline 2005 & 14 & 43 & 1093 & 314 & 1450 & 586 & 978 & -412 & 2198 & 4814 \\
\hline 2006 & -14 & 265 & 1152 & 395 & 1812 & 222 & 490 & 677 & 1388 & 4575 \\
\hline 2007 & -105 & 377 & -479 & 453 & 351 & -275 & 72 & 1862 & 4542 & 6448 \\
\hline 2008 & -65 & -825 & -468 & -347 & -1640 & 855 & 826 & -218 & 1237 & 993 \\
\hline
\end{tabular}

1 See chapter text. Estimates are given to the nearest $£$ million but cannot be 3 Wholesaling and retailing estimates exclude the motor trades.

regarded as accurate to this degree.

4 Quarterly alignment adjustment included in this series.

2 Components may not sum to totals due to rounding. 
1619 Gross fixed capital formation at current purchasers' prices: by broad sector United Kingdom. Total economy

\begin{tabular}{|c|c|c|c|c|c|c|c|c|c|c|}
\hline & & 2000 & 2001 & 2002 & 2003 & 2004 & 2005 & 2006 & 2007 & 2008 \\
\hline \multicolumn{11}{|l|}{ Private sector } \\
\hline New dwellings, excluding land & DFDF & 25604 & 27085 & 31455 & 34804 & 40927 & 43845 & 49273 & 51745 & 45910 \\
\hline Other buildings and structures & EQBU & 31966 & 32730 & 33580 & 35366 & 33227 & 36372 & 38047 & 44001 & . \\
\hline Transport equipment & EQBV & 12859 & 13897 & 15637 & 14708 & 13135 & 13818 & 14199 & 14714 & .. \\
\hline \multicolumn{11}{|l|}{ Other machinery and equipment } \\
\hline and cultivated assets & EQBW & 61236 & 58062 & 53498 & 50228 & 55138 & 56347 & 58339 & 64735 & .. \\
\hline Intangible fixed assets & $\mathrm{EQBX}$ & 4048 & 4285 & 4674 & 4894 & 5258 & 5594 & .. & .. & .. \\
\hline \multicolumn{10}{|l|}{ Costs associated with the transfer of } & 14211 \\
\hline Total & $\mathrm{EQBZ}$ & 146887 & 148756 & 154243 & 156385 & 170025 & 177123 & .. & .. & . \\
\hline \multicolumn{11}{|l|}{ Public non-financial corporations } \\
\hline New dwellings, excluding land & DEER & 1421 & 2387 & 2837 & 3509 & 3235 & 3574 & 4049 & 3899 & 4089 \\
\hline Other buildings and structures & DEES & 1775 & 1854 & 2304 & 2236 & 1493 & 2111 & 1830 & 1587 & . \\
\hline Transport equipment & DEEP & 178 & 171 & 110 & 126 & 193 & 334 & 181 & 175 & . \\
\hline \multicolumn{11}{|l|}{ Other machinery and equipment } \\
\hline \multicolumn{11}{|l|}{ Costs associated with the transfer of } \\
\hline ownership of non-produced assets & DLXQ & -2171 & -2254 & -2764 & -5674 & -5440 & -2675 & -2375 & -2032 & -1112 \\
\hline Total & FCCJ & 2354 & 3183 & 3830 & 1857 & 1260 & 20576 & 5440 & 5774 & 7435 \\
\hline \multicolumn{11}{|l|}{ General government } \\
\hline New dwellings, excluding land & DFHW & 369 & 334 & 207 & 149 & 137 & 71 & 9 & 31 & . \\
\hline Other buildings and structures & $\mathrm{EQCH}$ & 9434 & 10348 & 11678 & 14693 & 15866 & 18884 & 20930 & 22508 & 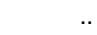 \\
\hline Transport equipment & EQ̃CI & 540 & 588 & 567 & 758 & 1011 & 610 & 500 & 499 & .. \\
\hline \multicolumn{11}{|l|}{ Other machinery and equipment } \\
\hline and cultivated assets & $E Q C J$ & 1699 & 2239 & 2867 & 3176 & 3652 & -12438 & 2480 & 2484 & . \\
\hline Intangible fixed assets & EQCK & 367 & 334 & 358 & 384 & 400 & 304 & 418 & 339 & .. \\
\hline \multicolumn{11}{|l|}{ Costs associated with the transfer of } \\
\hline ownership of non-produced assets & EQCL & -182 & -310 & -225 & 1349 & 2153 & -340 & -670 & -651 & . \\
\hline Total & NNBF & 12227 & 13533 & 15452 & 20509 & 23219 & 7091 & 23667 & 25542 & 33019 \\
\hline
\end{tabular}

1 See chapter text

Source: Office for National Statistics: 02070142083

2 Components may not sum to totals due to rounding.

Gross fixed capital formation at current purchasers' prices: by type of asset ${ }^{1,2}$ United Kingdom. Total economy

$£$ million

$\begin{array}{lllllllll}2000 & 2001 & 2002 & 2003 & 2004 & 2005 & 2006 & 2007 & 2008\end{array}$

Tangible fixed assets

New dwellings, excluding land

Other buildings and structures

Transport equipment

Other machinery and equipment

and cultivated assets

Total

Intangible fixed assets

$43175 \quad 44932$

DLWS

13577

14656

47562
16314

52295

50586

60807

68096

XI

63535

57152

54441

59832

60387

61805

68798

15020

EQCQ

$\overline{147681} \overline{150323} \quad \overline{155527} \overline{160790} \quad \overline{169056} \quad \overline{180006} \overline{190823} \quad \overline{209130}$

DLXP

$\overline{10670} \quad \overline{11326}$

12614

13850

14164

14387

15531

16045

Costs associated with the transfer of ownership of non-produced assets

DFBH

$8821 \quad 10133$

12410

12060

$17452 \quad 16925 \quad 21566 \quad 24053$

Total gross fixed capital formation

NPQX

167172

1 See chapter text.

2 Components may not sum to totals due to rounding.

Source: Office for National Statistics: 02070142083 
National accounts

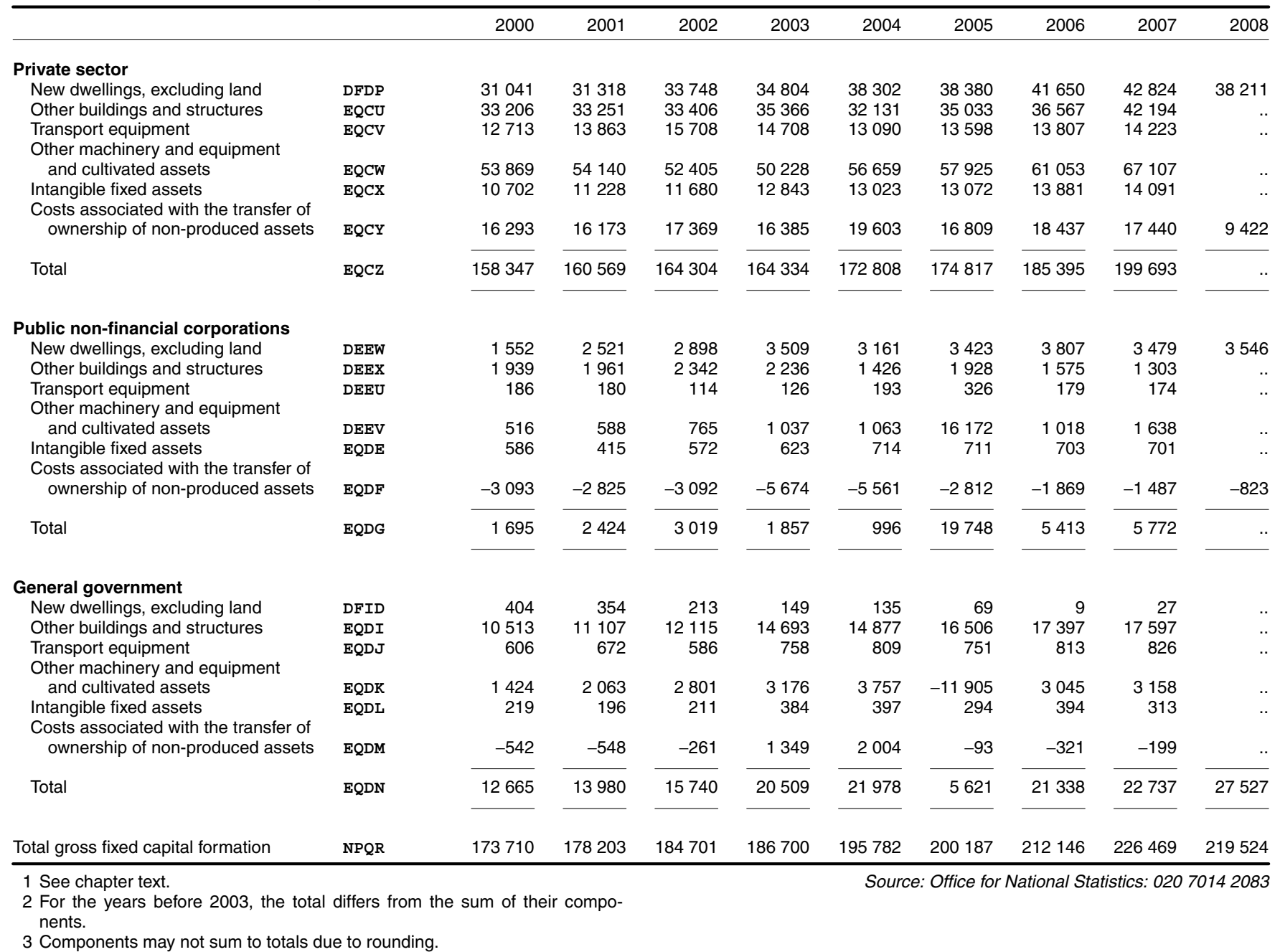

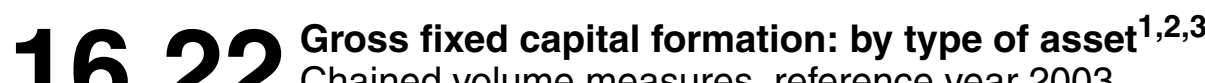 Chained volume measures, reference year 2003 United Kingdom. Total economy}

$£$ million

\begin{tabular}{lllllllll}
2000 & 2001 & 2002 & 2003 & 2004 & 2005 & 2006 & 2007 & 2008 \\
\hline
\end{tabular}

Tangible fixed assets

\begin{tabular}{|c|c|c|c|c|c|c|c|c|c|c|}
\hline New dwellings, excluding land & DFDV & 32888 & 34172 & 36839 & 38462 & 41598 & 41872 & 45466 & 46306 & 41770 \\
\hline Other buildings and structures & EQDP & 45780 & 46413 & 47913 & 52295 & 48434 & 53467 & 55539 & 61094 & \\
\hline Transport equipment & DLWJ & 13489 & 14698 & 16414 & 15592 & 14092 & 14675 & 14799 & 15211 & 14188 \\
\hline $\begin{array}{l}\text { Other machinery and equipment } \\
\text { and cultivated assets }\end{array}$ & DLWM & 55774 & 56780 & 55971 & 54441 & 61479 & 62192 & 65116 & 72252 & 70473 \\
\hline Total & EQDS & 148509 & 152571 & 157257 & 160790 & 165602 & 172205 & 180921 & 195763 & .. \\
\hline Intangible fixed assets & EQDT & 11445 & 11742 & 12371 & 13850 & 14134 & 14077 & 14978 & 15123 & 15520 \\
\hline $\begin{array}{l}\text { Costs associated with the transfer of } \\
\text { ownership of non-produced assets }\end{array}$ & DFDW & 12810 & 12960 & 14097 & 12060 & 16046 & 13904 & 16247 & 16319 & .. \\
\hline Total gross fixed capital formation & NPQR & 173710 & 178203 & 184701 & 186700 & 195782 & 200187 & 212146 & 226469 & 219524 \\
\hline
\end{tabular}

